\title{
Multiple periodic solutions for two classes of nonlinear difference systems involving classical $\left(\phi_{1}, \phi_{2}\right)$-Laplacian
}

\author{
Xingyong Zhang*, Liben Wang \\ Department of Mathematics, Faculty of Science, Kunming University of Science and Technology, Kunming, Yunnan 650500, P. R. China.
}

Communicated by A. Atangana

\begin{abstract}
In this paper, we investigate the existence of multiple periodic solutions for two classes of nonlinear difference systems involving $\left(\phi_{1}, \phi_{2}\right)$-Laplacian. First, by using an important critical point theorem due to B. Ricceri, we establish an existence theorem of three periodic solutions for the first nonlinear difference system with $\left(\phi_{1}, \phi_{2}\right)$-Laplacian and two parameters. Moreover, for the second nonlinear difference system with $\left(\phi_{1}, \phi_{2}\right)$-Laplacian, by using the Clark's Theorem, we obtain a multiplicity result of periodic solutions under a symmetric condition. Finally, two examples are given to verify our theorems. (C)2017 All rights reserved.
\end{abstract}

Keywords: Difference systems, periodic solutions, multiplicity, variational approach.

2010 MSC: 39A23, 37J45.

\section{Introduction and main results}

Let $\mathbb{R}$ denote the real numbers, $\mathbb{Z}$ the integer numbers, and given $a<b$ in $\mathbb{Z}$. Let $\mathbb{Z}[a, b]=\{a, a+$ $1, \ldots, b\}$. Let $T>1$ and $N$ be fixed positive integers.

Firstly, in this paper, we are concerned with the existence of three periodic solutions for the following nonlinear difference system:

$$
\left\{\begin{array}{l}
\mu \Delta\left[\rho_{1}(t-1) \phi_{1}\left(\Delta u_{1}(t-1)\right)\right]-\mu \rho_{3}(t) \phi_{3}\left(u_{1}(t)\right)+\nabla_{\mathfrak{u}_{1}} W\left(t, u_{1}(t), u_{2}(t)\right)=0 \\
\mu \Delta\left[\rho_{2}(t-1) \phi_{2}\left(\Delta u_{2}(t-1)\right)\right]-\mu \rho_{4}(t) \phi_{4}\left(u_{2}(t)\right)+\nabla_{\mathfrak{u}_{2}} W\left(t, u_{1}(t), u_{2}(t)\right)=0
\end{array}\right.
$$

where $\mu \in \mathbb{R}, \rho_{i}: \mathbb{R} \rightarrow \mathbb{R}^{+}, \phi_{i}, i=1,2,3,4$ satisfy the following conditions:

( $\rho) \rho_{i}$ are $T$-periodic and $\min _{t \in \mathbb{Z}[1, T]} \rho_{i}(t)>0, i=1,2,3,4$;

$(\mathcal{A} 1) \phi_{i}: \mathbb{R}^{N} \rightarrow \mathbb{R}^{N}$ are homeomorphisms such that $\phi_{i}(0)=0, \phi_{i}=\nabla \Phi_{i}$, with $\Phi_{i} \in C^{1}\left(\mathbb{R}^{N},[0,+\infty)\right)$ strictly convex and $\Phi_{i}(0)=0, i=1,2,3,4$.

\footnotetext{
*Corresponding author

Email address: zhangxingyong1@163.com (Xingyong Zhang)
}

doi:10.22436/jnsa.010.08.31 
Remark 1.1. Assumption $(\mathcal{A} 1)$ is given in [11], where it is used to characterize the classical homeomorphism.

Over the past ten years, variational methods have been used extensively to investigate the existence and multiplicity of solutions for difference systems (for example, see [1-6, 9, 11, 12, 19-22, 26-30]). In $[11,12]$, Mawhin investigated the following nonlinear discrete systems with $\phi$-Laplacian:

$$
\Delta \phi[\Delta \mathfrak{u}(\mathrm{n}-1)]=\nabla_{\mathfrak{u}} \mathrm{F}[\mathrm{n}, \mathrm{u}(\mathrm{n})]+\mathrm{h}(\mathrm{n}) \quad(\mathrm{n} \in \mathbb{Z}),
$$

where $\phi=\nabla \Phi$, and $\Phi$ strictly convex, is a homeomorphism of $\mathbb{R}^{N}$ onto the ball $B_{a} \subset \mathbb{R}^{N}$ or of $B_{a}$ onto $\mathbb{R}^{\mathrm{N}}$. The assumption about $\phi$ implies three cases: firstly, classical homeomorphism if $\phi: \mathbb{R}^{\mathrm{N}} \rightarrow$ $\mathbb{R}^{N}$, for example, $\phi(0)=0, \phi(x)=|x|^{p-2} x$ for some $p>1$ and all $x \in \mathbb{R}^{N} \backslash\{0\}$; secondly, bounded homeomorphism if $\phi: \mathbb{R}^{N} \rightarrow B_{a}(a<+\infty)$, for example, $\phi(x)=\frac{x}{\sqrt{1+|x|^{2}}} \in B_{1}$ for all $x \in \mathbb{R}^{N}$; finally, singular homeomorphism if $\phi: \mathrm{B}_{\mathrm{a}} \subset \mathbb{R}^{\mathrm{N}} \rightarrow \mathbb{R}^{\mathrm{N}}$, for example, $\phi(x)=\frac{x}{\sqrt{1-|x|^{2}}}$ for all $x \in \mathrm{B}_{1}$. Under some reasonable assumptions, by using variational methods, Mawhin obtained some existence and multiplicity results for system (1.2).

However, to the best of our knowledge, except for recent works in [3, 21, 29] which are made by our first author and his cooperators, there are no people to investigate the existence and multiplicity of solutions for system involving classical $\left(\phi_{1}, \phi_{2}\right)$-Laplacian. In [21], Wang and Zhang investigated the multiplicity of T-periodic solutions for the following nonlinear difference system:

$$
\left\{\begin{aligned}
\Delta \phi_{1}\left(\Delta \mathrm{u}_{1}(\mathrm{t}-1)\right) & =\nabla_{\mathrm{u}_{1}} \mathrm{~F}\left(\mathrm{t}, \mathrm{u}_{1}(\mathrm{t}), \mathrm{u}_{2}(\mathrm{t})\right)+\mathrm{h}_{1}(\mathrm{t}) \\
\Delta \phi_{2}\left(\Delta \mathrm{u}_{2}(\mathrm{t}-1)\right) & =\nabla_{\mathrm{u}_{2}} \mathrm{~F}\left(\mathrm{t}, \mathrm{u}_{1}(\mathrm{t}), \mathrm{u}_{2}(\mathrm{t})\right)+\mathrm{h}_{2}(\mathrm{t})
\end{aligned}\right.
$$

where $\mathrm{F}: \mathbb{Z} \times \mathbb{R}^{\mathrm{N}} \times \mathbb{R}^{\mathrm{N}} \rightarrow \mathbb{R}$ and $\phi_{\mathrm{m}}, \mathrm{m}=1,2$ satisfy the following condition:

(A) $\phi_{i}$ is a homeomrphism from $\mathbb{R}^{N}$ onto $\mathrm{B}_{\mathrm{a}} \subset \mathbb{R}^{\mathrm{N}}(\mathrm{a} \in(0,+\infty])$, such that $\phi_{i}(0)=0, \phi_{i}=\nabla \Phi_{i}$, with $\Phi_{i} \in C^{1}\left(\mathbb{R}^{N},[0,+\infty]\right)$ strictly convex and $\Phi_{i}(0)=0, m=1,2$.

Assumption $(\mathcal{A})$ implies that $\Phi_{i}, i=1,2$ are the classical homeomorphisms or the bounded homeomorphisms. They investigated the case that $F\left(t, x_{1}, x_{2}\right)$ is periodic on $r_{1}$ components of variables $x_{1}^{(1)}, \ldots, x_{N}^{(1)}$ and $r_{2}$ components of variables $x_{1}^{(2)}, \cdots, x_{N}^{(2)}$, where $1 \leqslant r_{1} \leqslant N$ and $1 \leqslant r_{2} \leqslant N$. By using a critical point theorem in [13] and a generalized saddle point theorem in [10], they obtained that system (1.3) has at least $r_{1}+r_{2}+1$ geometrically distinct $T$-periodic solutions. Their results generalize those results corresponding to classical homeomorphism and bounded homeomorphism in [12].

In [29], our first author and Wang investigated the existence of homoclinic solutions for the following nonlinear difference systems involving classical $\left(\phi_{1}, \phi_{2}\right)$-Laplacian:

$$
\left\{\begin{array}{l}
\Delta \phi_{1}\left(\Delta \mathrm{u}_{1}(\mathrm{t}-1)\right)+\nabla_{\mathfrak{u}_{1}} \mathrm{~V}\left(\mathrm{t}, \mathrm{u}_{1}(\mathrm{t}), \mathrm{u}_{2}(\mathrm{t})\right)=\mathrm{f}_{1}(\mathrm{t}) \\
\Delta \phi_{2}\left(\Delta \mathrm{u}_{2}(\mathrm{t}-1)\right)+\nabla_{\mathfrak{u}_{2}} \mathrm{~V}\left(\mathrm{t}, \mathrm{u}_{1}(\mathrm{t}), \mathrm{u}_{2}(\mathrm{t})\right)=\mathrm{f}_{2}(\mathrm{t})
\end{array}\right.
$$

where $t \in \mathbb{Z}, u_{m}(t) \in \mathbb{R}^{N}, m=1,2, V\left(t, x_{1}, x_{2}\right)=-K\left(t, x_{1}, x_{2}\right)+W\left(t, x_{1}, x_{2}\right), K, W: \mathbb{Z} \times \mathbb{R}^{N} \times \mathbb{R}^{N} \rightarrow \mathbb{R}$ and $\phi_{\mathrm{m}}, \mathrm{m}=1,2$ satisfy assumption $(\mathcal{A} 1)$. They first improved some inequalities in [7]. Then by using a linking theorem in [18], some new existence results of homoclinic solutions for system (1.4) were obtained when $W$ has super $p$-linear growth and $K$ has sub $p$-linear growth.

In [3], Deng et al. considered the existence of periodic solutions for the following $\left(\phi_{1}, \phi_{2}\right)$-Laplacian systems:

$$
\left\{\begin{array}{l}
\Delta \phi_{1}\left(\Delta \mathrm{u}_{1}(\mathrm{t}-1)\right)+\nabla_{\mathrm{u}_{1}} \mathrm{~F}\left(\mathrm{t}, \mathrm{u}_{1}(\mathrm{t}), \mathrm{u}_{2}(\mathrm{t})\right)=0, \\
\Delta \phi_{2}\left(\Delta \mathrm{u}_{2}(\mathrm{t}-1)\right)+\nabla_{\mathrm{u}_{2}} \mathrm{~F}\left(\mathrm{t}, \mathrm{u}_{1}(\mathrm{t}), \mathrm{u}_{2}(\mathrm{t})\right)=0 .
\end{array}\right.
$$

By using the saddle point theorem, they obtained some existence results for system with classical $\left(\phi_{1}, \phi_{2}\right)$ Laplacian if $F$ has $(p, q)$-sublinear growth, and some existence results for system with bounded $\left(\phi_{1}, \phi_{2}\right)$ Laplacian if $\mathrm{F}$ has sublinear growth. Moreover, by using the least action principle, they also obtained some 
existence results for system with classical or bounded $\left(\phi_{1}, \phi_{2}\right)$-Laplacian if $\mathrm{F}$ has a growth like Lipschitz condition.

Inspired by [11, 12, 17, 21, 29], in this paper, we are interested in the existence of three T-periodic solutions for system (1.1). By using an important three critical point theorem established by Ricceri in [17], we investigate the existence of three T-periodic solutions for system (1.1), as stated in the following.

We denote by $\mathcal{C}$, the class of all functions $F: \mathbb{Z} \times \mathbb{R}^{N} \times \mathbb{R}^{N} \longrightarrow \mathbb{R}^{N}$ such that $\mathrm{F}\left(\cdot, x_{1}, x_{2}\right)$ is T-periodic in $\mathbb{Z}$ for all $\left(x_{1}, x_{2}\right) \in \mathbb{R}^{N} \times \mathbb{R}^{N}$ and continuously differentiable in $\mathbb{R}^{N} \times \mathbb{R}^{N}$ for all $t \in \mathbb{Z}[1, T]$. Define

$$
\mathrm{E}_{\mathrm{T}}=\left\{\mathrm{h}:=\{\mathrm{h}(\mathrm{t})\}_{\mathrm{t} \in \mathbb{Z}} \mid \mathrm{h}(\mathrm{t}+\mathrm{T})=\mathrm{h}(\mathrm{t}), \mathrm{h}(\mathrm{t}) \in \mathbb{R}^{\mathrm{N}}, \mathrm{t} \in \mathbb{Z}\right\}
$$

and let $E=E_{T} \times E_{T}$. Then $E$ is finite-dimensional. On $E_{T}$, we define

$$
\|h\|_{E_{T}}=\left(\sum_{t=1}^{T}|\Delta h(t)|^{\theta}+\sum_{t=1}^{T}|h(t)|^{\theta}\right)^{1 / \theta} .
$$

For $u=\left(u_{1}, u_{2}\right) \in E$, define

$$
\|\mathrm{u}\|=\left\|\mathrm{u}_{1}\right\|_{\mathrm{E}_{\mathrm{T}}}+\left\|\mathrm{u}_{2}\right\|_{\mathrm{E}_{\mathrm{T}}} .
$$

Define

$$
\begin{aligned}
I(u) & =\sum_{t=1}^{T}\left[\rho_{1}(t) \Phi_{1}\left(\Delta u_{1}(t)\right)+\rho_{2}(t) \Phi_{2}\left(\Delta u_{2}(t)\right)+\rho_{3}(t) \Phi_{3}\left(u_{1}(t)\right)+\rho_{4}(t) \Phi_{4}\left(u_{2}(t)\right)\right] \\
\Psi(u) & =-\sum_{t=1}^{T} F\left(t, u_{1}(t), u_{2}(t)\right), \\
\Phi(u) & =\sum_{t=1}^{T} G\left(t, u_{1}(t), u_{2}(t)\right), \\
\Gamma(u) & =-\sum_{t=1}^{T} H\left(t, u_{1}(t), u_{2}(t)\right), \quad u \in E
\end{aligned}
$$

where $F, G, H$ are functions belonging to $\mathcal{C}$.

Theorem 1.2. Let $\mathrm{F}, \mathrm{G} \in \mathcal{C}$ and suppose that $(\rho),(\mathcal{A} 1)$ and the following conditions hold:

$(\mathcal{A} 2)$ there exist positive constants $\mathrm{c}_{\mathfrak{i}}(i=1,2,3,4), \theta>1$ such that

$$
\left(\phi_{i}(x)-\phi_{i}(y), x-y\right) \geqslant c_{i}|x-y|^{\theta}, \quad \forall x, y \in \mathbb{R}^{N}, i=1,2,3,4,
$$

where $(\cdot, \cdot)$ stands for the usual product in $\mathbb{R}^{\mathrm{N}}$;

(A3) $\lim _{|x| \rightarrow \infty} \Phi_{i}(x)=+\infty$ and there exist positive constants $l \geqslant \theta, d_{i}$ and $m_{i}$ such that $\Phi_{i}(x) \leqslant d_{i}|x|^{l}+m_{i}$ for all $x \in \mathbb{R}^{\mathrm{N}},(i=1,2,3,4)$;

(A4) for all $t \in \mathbb{Z}[1, T]$ and all $\lambda>0$, there exists $C_{0}(\lambda) \in \mathbb{R}$ such that for all $\left(x_{1}, x_{2}\right) \in \mathbb{R}^{N} \times \mathbb{R}^{N}$,

$$
\lim _{\left|x_{1}\right|+\left|x_{2}\right| \rightarrow \infty} \frac{F\left(t, x_{1}, x_{2}\right)}{\left|x_{1}\right|^{l}+\left|x_{2}\right|^{l}}=+\infty, \quad \lambda G\left(t, x_{1}, x_{2}\right) \geqslant F\left(t, x_{1}, x_{2}\right)+C_{0}(\lambda)
$$

(A5) $\sum_{\mathrm{t}=1}^{\mathrm{T}} \mathrm{G}(\mathrm{t}, 0,0)=0$.

Then for each $r>0, \mu>\max \left\{0, \mu^{*}(I, \Psi, \Phi, r)\right\}$, and compact interval $\left.[a, b] \subset\right] 0, \beta(\mu \mathrm{I}+\Psi, \Phi, r)[$, there exists a number $\rho>0$ with the following property: for every $\lambda \in[a, b]$ and every $\mathrm{H} \in \mathcal{C}$, there exists $\delta>0$ such that, 
for each $v \in[0, \delta]$, system (1.1) has at least three T-periodic solutions in $\mathrm{E}$ whose norms are less than $\rho$, where $W=F-\lambda G+v H$,

$$
\begin{aligned}
& \beta(\mu \mathrm{I}+\Psi, \Phi, r)=\sup _{u \in \Phi^{-1}(] r,+\infty[)} \frac{\mu \mathrm{I}(u)+\Psi(u)-\inf _{\left.\left.\Phi^{-1}(]-\infty, r\right]\right)}(\mu \mathrm{I}+\Psi)}{r-\Phi(u)}, \\
& \mu^{*}(I, \Psi, \Phi, r)=\inf \left\{\frac{\Psi(u)-\gamma+r}{\eta_{r}-I(u)}: u \in E, \Phi(u)<r, I(u)<\eta_{r}\right\}, \\
& \gamma=\inf _{E}(\Psi(u)+\Phi(u)), \quad \eta_{r}=\inf _{u \in \Phi^{-1}(r)} I(u) .
\end{aligned}
$$

Inspired by [17], we have the following corollary:

Corollary 1.3. Suppose that $(\rho),(\mathcal{A} 1)-(\mathcal{A} 3)$ and $(\mathcal{A} 5)$ hold. If

$(\mathcal{A} 4)^{\prime}$ there exists $\mathrm{s}>\mathrm{l}$ such that for every $\mathrm{t} \in \mathbb{Z}[1, \mathrm{~T}]$,

$$
\lim _{\left|x_{1}\right|+\left|x_{2}\right| \rightarrow \infty} \frac{F\left(t, x_{1}, x_{2}\right)}{\left|x_{1}\right|^{l}+\left|x_{2}\right|^{l}}=+\infty, \lim _{\left|x_{1}\right|+\left|x_{2}\right| \rightarrow \infty} \frac{F\left(t, x_{1}, x_{2}\right)}{\left|x_{1}\right|^{s}+\left|x_{2}\right|^{s}}<+\infty, \text { and } \lim _{\left|x_{1}\right|+\left|x_{2}\right| \rightarrow \infty} \frac{G\left(t, x_{1}, x_{2}\right)}{\left|x_{1}\right|^{s}+\left|x_{2}\right|^{s}}=+\infty,
$$

then the conclusion of Theorem 1.2 holds.

Moreover, in this paper, we are also concerned with the multiplicity of T-periodic solutions for the following nonlinear difference system:

$$
\left\{\begin{array}{l}
\Delta\left(\gamma_{1}(t-1) \phi_{1}\left(\Delta \mathfrak{u}_{1}(t-1)\right)\right)-\gamma_{3}(t) \phi_{3}\left(\left|u_{1}(t)\right|\right)+\nabla_{\mathfrak{u}_{1}} \mathrm{~F}\left(\mathrm{t}, \mathrm{u}_{1}(\mathrm{t}), \mathrm{u}_{2}(\mathrm{t})\right)=0 \\
\Delta\left(\gamma_{2}(\mathrm{t}-1) \phi_{2}\left(\Delta \mathrm{u}_{2}(\mathrm{t}-1)\right)\right)-\gamma_{4}(\mathrm{t}) \phi_{4}\left(\left|\mathrm{u}_{2}(\mathrm{t})\right|\right)+\nabla_{\mathfrak{u}_{2}} \mathrm{~F}\left(\mathrm{t}, \mathrm{u}_{1}(\mathrm{t}), \mathrm{u}_{2}(\mathrm{t})\right)=0
\end{array}\right.
$$

where $\gamma_{i}: \mathbb{R} \rightarrow \mathbb{R}^{+}$satisfy the following conditions:

$(\gamma) \gamma_{i}$ are T-periodic and $\min _{t \in \mathbb{Z}[1, T]} \gamma_{i}(t)>0, i=1,2,3,4$,

and $\phi_{i}, i=1,2,3,4$ satisfy the assumption $(\mathcal{A} 1)$ and the following condition:

$(\phi)$ there exist positive constants $p>1, q>1, a_{i}, b_{i}, i=1,2,3,4$ such that

$$
a_{i}|x|^{q} \leqslant \Phi_{i}(x) \leqslant b_{i}|x|^{q}, i=1,3, \quad \forall x \in \mathbb{R}^{N}
$$

and

$$
a_{i}|x|^{p} \leqslant \Phi_{i}(x) \leqslant b_{i}|x|^{p}, i=2,4, \quad \forall x \in \mathbb{R}^{N} .
$$

Moreover, $\mathrm{F}: \mathbb{Z} \times \mathbb{R}^{N} \times \mathbb{R}^{N} \longrightarrow \mathbb{R},\left(t, x_{1}, x_{2}\right) \longrightarrow F\left(t, x_{1}, x_{2}\right)$ is T-periodic in $t$ for all $\left(x_{1}, x_{2}\right) \in \mathbb{R}^{N} \times \mathbb{R}^{N}$ and continuously differentiable in $\left(x_{1}, x_{2}\right)$ for every $t \in \mathbb{Z}[1, T]$.

When $\Phi_{i}(x)=\frac{1}{q}|x|^{q}, i=1,3$ and $\Phi_{i}(x)=\frac{1}{p}|x|^{p}, i=2,4$, system (1.5) can be seen as a discrete analogue of the following $(q, p)$-Laplacian differential systems:

$$
\left\{\begin{array}{l}
\frac{d\left(\gamma_{1}(t)\left|\dot{u}_{1}(t)\right|^{q-2} \dot{u}_{1}(t)\right)}{d t}-\gamma_{3}(t)\left|u_{1}(t)\right|^{q-2} u_{1}(t)+\nabla_{\mathfrak{u}_{1}} F\left(t, u_{1}(t), u_{2}(t)\right)=0 \\
\frac{d\left(\gamma_{2}(t)\left|\dot{u}_{2}(t)\right|^{p-2} \dot{u}_{2}(t)\right)}{d t}-\gamma_{4}(t)\left|u_{2}(t)\right|^{p-2} u_{2}(t)+\nabla_{u_{2}} F\left(t, u_{1}(t), u_{2}(t)\right)=0
\end{array}\right.
$$

Recently, by using variational methods, system (1.6) has been investigated by some authors (for example, see $[8,14,15,23,24]$,$) and some interesting results on the existence and multiplicity of solutions have$ been obtained. However, to the best of our knowledge, there are no people to investigate the nonlinear difference system (1.5). In this paper, inspired by $[8,14,15,19,23,24]$, we are interested in the existence and multiplicity of T-periodic solutions for system (1.5). By using the Clark's theorem, we obtain the following theorem. 
Theorem 1.4. Suppose that $(\gamma),(\phi)$, and the following conditions hold:

$(\mathcal{F} 0)$ there exist $\alpha_{1} \in[0, q), \alpha_{2} \in[0, p), h_{i}: \mathbb{Z}[1, T] \rightarrow \mathbb{R}^{+}, i=1,2$ and $l: \mathbb{Z}[1, T] \rightarrow \mathbb{R}^{+}$such that

$$
F\left(t, x_{1}, x_{2}\right) \leqslant h_{1}(t)\left|x_{1}\right|^{\alpha_{1}}+h_{2}(t)\left|x_{2}\right|^{\alpha_{2}}+l(t) ;
$$

(F1) $\mathrm{F}(\mathrm{t}, 0,0)=0$;

(F2) $\mathrm{F}\left(\mathrm{t},-\mathrm{x}_{1},-\mathrm{x}_{2}\right)=\mathrm{F}\left(\mathrm{t}, \mathrm{x}_{1}, \mathrm{x}_{2}\right)$;

(F3) there exist constants $\beta_{i} \in(1, \min \{q, p\}), M_{i} \in(0, \infty), i=1,2$ and $\delta \in(0,1)$ such that

$$
\mathrm{F}\left(\mathrm{t}, \mathrm{x}_{1}, \mathrm{x}_{2}\right) \geqslant \mathrm{M}_{1}\left|\mathrm{x}_{1}\right|^{\beta_{1}}+\mathrm{M}_{2}\left|\mathrm{x}_{2}\right|^{\beta_{2}}, \quad \forall\left|x_{1}\right|<\delta,\left|x_{2}\right|<\delta .
$$

Then system (1.5) has at least 2NT distinct pairs of nonzero solutions.

\section{Preliminaries}

For $h \in E_{T}$, set

$$
\|h\|_{r}=\left(\sum_{t=1}^{T}|h(t)|^{r}\right)^{1 / r} \text { and }\|h\|_{\infty}=\max _{t \in \mathbb{Z}[1, T]}|h(t)|, \quad r>1 .
$$

Obviously, we have

$$
\|h\|_{\infty} \leqslant\|h\|_{r} \leqslant T^{1 / r}\|h\|_{\infty}
$$

On $E_{T}$, define

$$
\|h\|_{\left[E_{T}\right]}=\left(\sum_{t=1}^{T}|\Delta h(t)|^{l}+\sum_{t=1}^{T}|h(t)|^{l}\right)^{1 / l} .
$$

Note that $E_{T}$ is finite-dimensional. $\|\cdot\|_{E_{T}}$ is equivalent to $\|\cdot\|_{r}(r>1)$ and $\|\cdot\|_{\left[E_{T}\right]}$. Hence, there exist positive constants $C_{i}(i=1, \cdots, 6)$ such that

$$
\begin{aligned}
& C_{1}\|\cdot\|_{\mathrm{E}_{\mathrm{T}}} \leqslant\|\cdot\|_{\theta} \leqslant \mathrm{C}_{2}\|\cdot\|_{\mathrm{E}_{\mathrm{T}},} \\
& \mathrm{C}_{3}\|\cdot\|_{\mathrm{E}_{\mathrm{T}}} \leqslant\|\cdot\|_{\mathrm{l}} \leqslant \mathrm{C}_{4}\|\cdot\|_{\mathrm{E}_{\mathrm{T}},} \\
& \mathrm{C}_{5}\|\cdot\|_{\mathrm{E}_{\mathrm{T}}} \leqslant\|\cdot\|_{\left[\mathrm{E}_{\mathrm{T}}\right]} \leqslant \mathrm{C}_{6}\|\cdot\|_{\mathrm{E}_{\mathrm{T}}} .
\end{aligned}
$$

Lemma 2.1 ([21]). Let $\mathrm{L}: \mathbb{Z}[1, \mathrm{~T}] \times \mathbb{R}^{\mathrm{N}} \times \mathbb{R}^{\mathrm{N}} \times \mathbb{R}^{\mathrm{N}} \times \mathbb{R}^{\mathrm{N}} \longrightarrow \mathbb{R},\left(\mathrm{t}, \mathrm{x}_{1}, \mathrm{x}_{2}, \mathrm{y}_{1}, \mathrm{y}_{2}\right) \longrightarrow \mathrm{L}\left(\mathrm{t}, \mathrm{x}_{1}, \mathrm{x}_{2}, \mathrm{y}_{1}, \mathrm{y}_{2}\right)$ and assume that $\mathrm{L}$ is continuously differentiable in $\left(\mathrm{x}_{1}, \mathrm{x}_{2}, \mathrm{y}_{1}, \mathrm{y}_{2}\right)$ for all $\mathrm{t} \in \mathbb{Z}[1, \mathrm{~T}]$. Then the functional $\varphi: \mathrm{E} \rightarrow \mathbb{R}$ defined by

$$
\varphi(u)=\varphi\left(u_{1}, u_{2}\right)=\sum_{t=1}^{T} L\left(t, u_{1}(t), u_{2}(t), \Delta u_{1}(t), \Delta u_{2}(t)\right)
$$

is continuously differentiable on $\mathrm{E}$ and for $\mathrm{u}, v \in \mathrm{E}$,

$$
\begin{aligned}
\left\langle\varphi^{\prime}(\mathrm{u}), v\right\rangle=\left\langle\varphi^{\prime}\left(\mathrm{u}_{1}, \mathrm{u}_{2}\right),\left(v_{1}, v_{2}\right)\right\rangle= & \sum_{\mathrm{t}=1}^{\mathrm{T}}\left[\left(\mathrm{D}_{\mathrm{x}_{1}} \mathrm{~L}\left(\mathrm{t}, \mathrm{u}_{1}(\mathrm{t}), \mathrm{u}_{2}(\mathrm{t}), \Delta \mathrm{u}_{1}(\mathrm{t}), \Delta \mathrm{u}_{2}(\mathrm{t})\right), v_{1}(\mathrm{t})\right)\right. \\
& +\left(\mathrm{D}_{\mathrm{y}_{1}} \mathrm{~L}\left(\mathrm{t}, \mathrm{u}_{1}(\mathrm{t}), \mathrm{u}_{2}(\mathrm{t}), \Delta \mathrm{u}_{1}(\mathrm{t}), \Delta \mathrm{u}_{2}(\mathrm{t})\right), \Delta v_{1}(\mathrm{t})\right) \\
& +\left(\mathrm{D}_{\mathrm{x}_{2}} \mathrm{~L}\left(\mathrm{t}, \mathrm{u}_{1}(\mathrm{t}), \mathrm{u}_{2}(\mathrm{t}), \Delta \mathrm{u}_{1}(\mathrm{t}), \Delta \mathrm{u}_{2}(\mathrm{t})\right), v_{2}(\mathrm{t})\right) \\
& \left.+\left(\mathrm{D}_{\mathrm{y}_{2}} \mathrm{~L}\left(\mathrm{t}, \mathrm{u}_{1}(\mathrm{t}), \mathrm{u}_{2}(\mathrm{t}), \Delta \mathrm{u}_{1}(\mathrm{t}), \Delta \mathrm{u}_{2}(\mathrm{t})\right), \Delta v_{2}(\mathrm{t})\right)\right] .
\end{aligned}
$$

Let

$$
\begin{aligned}
\mathrm{L}\left(\mathrm{t}, \mathrm{x}_{1}, \mathrm{x}_{2}, \mathrm{y}_{1}, \mathrm{y}_{2}\right)= & \mu\left[\rho_{1}(\mathrm{t}) \Phi_{1}\left(\mathrm{y}_{1}\right)+\rho_{2}(\mathrm{t}) \Phi_{2}\left(\mathrm{y}_{2}\right)+\rho_{3}(t) \Phi_{3}\left(\mathrm{x}_{1}\right)+\rho_{4}(\mathrm{t}) \Phi_{4}\left(\mathrm{x}_{2}\right)\right] \\
& -\mathrm{F}\left(\mathrm{t}, \mathrm{x}_{1}, \mathrm{x}_{2}\right)+\lambda \mathrm{G}\left(\mathrm{t}, \mathrm{x}_{1}, \mathrm{x}_{2}\right)-v \mathrm{H}\left(\mathrm{t}, \mathrm{x}_{1}, \mathrm{x}_{2}\right),
\end{aligned}
$$

where $F, G, H: \mathbb{Z}[1, T] \times \mathbb{R}^{N} \times \mathbb{R}^{N} \longrightarrow \mathbb{R}^{N}$ are continuously differentiable in $\left(x_{1}, x_{2}\right) \in \mathbb{R}^{N} \times \mathbb{R}^{N}$ for all 
$t \in \mathbb{Z}[1, T]$. Then

$$
\begin{aligned}
\varphi(u)= & \sum_{t=1}^{T}\left[\mu\left(\rho_{1}(t) \Phi_{1}\left(\Delta u_{1}(t)\right)+\rho_{2}(t) \Phi_{2}\left(\Delta u_{2}(t)\right)+\rho_{3}(t) \Phi_{3}\left(u_{1}(t)\right)+\rho_{4}(t) \Phi_{4}\left(u_{2}(t)\right)\right)\right. \\
& \left.-F\left(t, u_{1}(t), u_{2}(t)\right)+\lambda G\left(t, u_{1}(t), u_{2}(t)\right)-v H\left(t, u_{1}(t), u_{2}(t)\right)\right] .
\end{aligned}
$$

Obviously, when $(\mathcal{A} 1)$ and $(\mathcal{A} 2)$ hold, $\varphi$ is continuously differentiable on $\mathrm{E}$ and for all $u, v \in \mathrm{E}$, we have

$$
\begin{aligned}
& \left\langle\varphi^{\prime}(u), v\right\rangle=\left\langle\varphi^{\prime}\left(u_{1}, u_{2}\right),\left(v_{1}, v_{2}\right)\right\rangle \\
& =\sum_{t=1}^{T}\left[\mu \rho_{1}(t)\left(\phi_{1}\left(\Delta u_{1}(t)\right), \Delta v_{1}(t)\right)+\mu \rho_{2}(t)\left(\phi_{2}\left(\Delta u_{2}(t)\right), \Delta v_{2}(t)\right)\right. \\
& \left.+\mu \rho_{3}(t)\left(\phi_{3}\left(u_{1}(t)\right), v_{1}(t)\right)+\mu \rho_{4}(t)\left(\phi_{4}\left(u_{2}(t)\right), v_{2}(t)\right)\right] \\
& -\sum_{\mathrm{t}=1}^{\mathrm{T}}\left[\left(\nabla_{\mathrm{u}_{1}} \mathrm{~F}\left(\mathrm{t}, \mathrm{u}_{1}(\mathrm{t}), \mathrm{u}_{2}(\mathrm{t})\right), v_{1}(\mathrm{t})\right)+\left(\nabla_{\mathrm{u}_{2}} \mathrm{~F}\left(\mathrm{t}, \mathrm{u}_{1}(\mathrm{t}), \mathrm{u}_{2}(\mathrm{t})\right), v_{2}(\mathrm{t})\right)\right] \\
& +\lambda \sum_{\mathrm{t}=1}^{\mathrm{T}}\left[\left(\nabla_{\mathrm{u}_{1}} \mathrm{G}\left(\mathrm{t}, \mathrm{u}_{1}(\mathrm{t}), \mathrm{u}_{2}(\mathrm{t})\right), v_{1}(\mathrm{t})\right)+\left(\nabla_{\mathrm{u}_{2}} \mathrm{G}\left(\mathrm{t}, \mathrm{u}_{1}(\mathrm{t}), \mathrm{u}_{2}(\mathrm{t})\right), v_{2}(\mathrm{t})\right)\right] \\
& -v \sum_{t=1}^{T}\left[\left(\nabla_{\mathfrak{u}_{1}} \mathrm{H}\left(\mathrm{t}, \mathrm{u}_{1}(\mathrm{t}), \mathrm{u}_{2}(\mathrm{t})\right), v_{1}(\mathrm{t})\right)+\left(\nabla_{\mathbf{u}_{2}} \mathrm{H}\left(\mathrm{t}, \mathrm{u}_{1}(\mathrm{t}), \mathrm{u}_{2}(\mathrm{t})\right), v_{2}(\mathrm{t})\right)\right] \text {. }
\end{aligned}
$$

Lemma 2.2. If $u \in E$ is a solution of Euler equation $\varphi^{\prime}(u)=0$, then $u$ is a solution of system (1.1).

Proof. At first, for any $u=\left(u_{1}, u_{2}\right), v=\left(v_{1}, v_{2}\right) \in E$, we can obtain the following two equalities:

$$
\begin{aligned}
& -\sum_{t=1}^{T}\left(\Delta\left[\rho_{1}(t-1) \phi_{1}\left(\Delta u_{1}(t-1)\right)\right], v_{1}(t)\right)=\sum_{t=1}^{T}\left(\rho_{1}(t) \phi_{1}\left(\Delta u_{1}(t)\right), \Delta v_{1}(t)\right), \\
& -\sum_{t=1}^{T}\left(\Delta\left[\rho_{2}(t-1) \phi_{2}\left(\Delta u_{2}(t-1)\right)\right], v_{2}(t)\right)=\sum_{t=1}^{T}\left(\rho_{2}(t) \phi_{2}\left(\Delta u_{2}(t)\right), \Delta v_{2}(t)\right) .
\end{aligned}
$$

In fact, since $u_{1}(t)=u_{1}(t+T)$ and $v_{1}(t)=v_{1}(t+T)$ for all $t \in \mathbb{Z}$, then

$$
\begin{aligned}
-\sum_{t=1}^{T} & \left(\Delta\left[\rho_{1}(t-1) \phi_{1}\left(\Delta u_{1}(t-1)\right)\right], v_{1}(t)\right) \\
& =-\sum_{t=1}^{T}\left(\rho_{1}(t) \phi_{1}\left(\Delta u_{1}(t)\right), v_{1}(t)\right)+\sum_{t=1}^{T}\left(\rho_{1}(t-1) \phi_{1}\left(\Delta u_{1}(t-1)\right), v_{1}(t)\right) \\
& =-\sum_{t=1}^{T}\left(\rho_{1}(t) \phi_{1}\left(\Delta u_{1}(t)\right), v_{1}(t)\right)+\sum_{t=1}^{T-1}\left(\rho_{1}(t) \phi_{1}\left(\Delta u_{1}(t)\right), v_{1}(t+1)\right)+\left(\rho_{1}(0) \phi_{1}\left(\Delta u_{1}(0)\right), v_{1}(1)\right) \\
& =\sum_{t=1}^{T}\left(\rho_{1}(t) \phi_{1}\left(\Delta u_{1}(t)\right), \Delta v_{1}(t)\right)+\left(\rho_{1}(0) \phi_{1}\left(\Delta u_{1}(0)\right), v_{1}(1)\right)-\left(\rho_{1}(T) \phi_{1}\left(\Delta u_{1}(T)\right), v_{1}(T+1)\right) \\
& =\sum_{t=1}^{T}\left(\rho_{1}(t) \phi_{1}\left(\Delta u_{1}(t)\right), \Delta v_{1}(t)\right) .
\end{aligned}
$$

Hence, (2.6) holds. Similarly, it is easy to get (2.7). Since $\varphi^{\prime}(u)=0$, then for all $v=\left(v_{1}, 0\right) \in \mathrm{E},(2.5)$ implies 
that

$$
\begin{aligned}
\sum_{t=1}^{T}\left[\mu\left(\rho_{1}(t) \phi_{1}\left(\Delta u_{1}(t)\right), \Delta v_{1}(t)\right)+\mu\left(\rho_{3}(t) \phi_{3}\left(u_{1}(t)\right), v_{1}(t)\right)\right] \\
=\sum_{t=1}^{T}\left(\nabla_{\mathfrak{u}_{1}} F\left(t, u_{1}(t), u_{2}(t)\right), v_{1}(t)\right)-\lambda \sum_{t=1}^{T}\left(\nabla_{\mathfrak{u}_{1}} G\left(t, u_{1}(t), u_{2}(t)\right), v_{1}(t)\right) \\
\quad+v \sum_{t=1}^{T}\left(\nabla_{\mathfrak{u}_{1}} H\left(t, u_{1}(t), u_{2}(t)\right), v_{1}(t)\right) .
\end{aligned}
$$

Note that $v_{1}$ is arbitrary. Then (2.6) and (2.8) imply that

$$
\mu \Delta\left[\rho_{1}(t-1) \phi_{1}\left(\Delta \mathfrak{u}_{1}(t-1)\right)\right]-\mu \rho_{3}(t) \phi_{3}\left(\mathfrak{u}_{1}(t)\right)+\nabla_{\mathfrak{u}_{1}} W\left(t, u_{1}(t), u_{2}(t)\right)=0 .
$$

Similarly, let $v_{1}=0$. We can obtain that

$$
\mu \Delta\left[\rho_{2}(t-1) \phi_{2}\left(\Delta \mathfrak{u}_{2}(t-1)\right)\right]-\mu \rho_{4}(t) \phi_{4}\left(u_{2}(t)\right)+\nabla_{\mathfrak{u}_{2}} W\left(t, u_{1}(t), u_{2}(t)\right)=0 .
$$

To prove Theorem 1.2, we will use the following three critical points theorem due to Ricceri [17].

Theorem 2.3 ([17]). Let $\mathrm{X}$ be a reflexive real Banach space, I :X $\rightarrow \mathbb{R}$ a sequentially weakly lower semicontinuous, coercive, bounded on each bounded subset of $\mathrm{X}, \mathrm{C}^{1}$ functional whose derivative admits a continuous inverse on $\mathrm{X}^{*}$, and $\Psi, \Phi: X \rightarrow \mathbb{R}$ two $C^{1}$ functionals with compact derivative. Assume also that the functional $\Psi+\lambda \Phi$ is bounded below for all $\lambda>0$ and that

$$
\liminf _{\|x\| \rightarrow+\infty} \frac{\Psi(x)}{\mathrm{I}(x)}=-\infty
$$

Then, for each $r>\sup _{M} \Phi$, where $M$ is the set of all global minima of $I$, for each $\mu>\max \left\{0, \mu^{*}(I, \Psi, \Phi, r)\right\}$, and for each compact interval $[a, b] \subset] 0, \beta(\mu \mathrm{I}+\Psi, \Phi, r)[$, there exists a number $\rho>0$ with the following property: for every $\lambda \in[\mathrm{a}, \mathrm{b}]$ and every $\mathrm{C}^{1}$ functional $\Gamma: \mathrm{X} \rightarrow \mathbb{R}$ with compact derivative, there exists $\delta>0$ such that, for each $v \in[0, \delta]$, the equation

$$
\mu \mathrm{I}^{\prime}(\mathrm{x})+\Psi^{\prime}(\mathrm{x})+\lambda \Phi^{\prime}(\mathrm{x})+v \Gamma^{\prime}(\mathrm{x})=0
$$

has at least three solutions in $\mathrm{X}$ whose norms are less than $\rho$, where

$$
\begin{aligned}
\beta(\mu \mathrm{I}+\Psi, \Phi, r) & =\sup _{x \in \Phi^{-1}(] \mathrm{r},+\infty[)} \frac{\mu \mathrm{I}(x)+\Psi(x)-\inf _{\left.\left.\Phi^{-1}(]-\infty, r\right]\right)}(\mu \mathrm{I}+\Psi)}{r-\Phi(x)}, \\
\mu^{*}(\mathrm{I}, \Psi, \Phi, r) & =\inf \left\{\frac{\Psi(x)-\gamma+r}{\eta_{r}-I(x)}: x \in X, \Phi(x)<r, I(x)<\eta_{r}\right\} \\
\gamma & =\inf _{X}(\Psi(x)+\Phi(x)), \quad \eta_{r}=\inf _{x \in \Phi^{-1}(r)} I(x) .
\end{aligned}
$$

\section{Proof of Theorem 1.2}

For the sake of convenience, we denote

$$
\rho_{i}^{+}=\max _{t \in \mathbb{Z}[1, T]} \rho_{i}(t), \quad \rho_{i}^{-}=\min _{t \in \mathbb{Z}[1, T]} \rho_{i}(t), \quad i=1,2,3,4 .
$$

Proof of Theorem 1.2. We prove that $\varphi$ defined by (2.4) satisfies all the assumptions of Theorem 2.3. Let $X=E$. Then $E$ is a reflexive and separable Banach space. Since all the vector topologies are equivalent in 
the finite dimensional Banach space $E$, then for any sequence $\left\{u^{\mathfrak{n}}\right\} \subset E$, assume that

$$
\mathrm{u}^{\mathrm{n}} \rightarrow \mathrm{u}^{*} \text { in } \mathrm{E} \text { as } \mathrm{n} \rightarrow \infty,
$$

that is,

$$
\begin{aligned}
& \left(\sum_{t=1}^{T}\left|\Delta u_{1}^{n}(t)-\Delta u_{1}^{*}(t)\right|^{\theta}+\sum_{t=1}^{T}\left|u_{1}^{n}(t)-u_{1}^{*}(t)\right|^{\theta}\right)^{1 / \theta}+\left(\sum_{t=1}^{T}\left|\Delta u_{2}^{n}(t)-\Delta u_{2}^{*}(t)\right|^{\theta}+\sum_{t=1}^{T}\left|u_{2}^{n}(t)-u_{2}^{*}(t)\right|^{\theta}\right)^{1 / \theta} \\
& \quad=\left\|u^{n}-u^{*}\right\| \rightarrow 0, \text { as } n \rightarrow \infty
\end{aligned}
$$

which implies that $\lim _{n \rightarrow \infty}\left|\Delta u_{i}^{n}(t)-\Delta u_{i}^{*}(t)\right|=0$ and $\lim _{n \rightarrow \infty}\left|u_{i}^{n}(t)-u_{i}^{*}(t)\right|=0, i=1,2$ for every $t \in \mathbb{Z}[1, T]$. Hence, it is easy to obtain that $(\mathcal{A} 1)$ implies that $\mathrm{I}$ is continuous in $\mathrm{E}$ and then sequentially weakly lower semicontinuous. Moreover, obviously, $(\mathcal{A} 1)$ and Lemma 2.1 imply that $\mathrm{I}$ is a $\mathrm{C}^{1}$ functional and

$$
\begin{aligned}
\left\langle I^{\prime}(u), v\right\rangle= & \sum_{t=1}^{T}\left[\rho_{1}(t)\left(\phi_{1}\left(\Delta u_{1}(t)\right), \Delta v_{1}(t)\right)+\rho_{2}(t)\left(\phi_{2}\left(\Delta u_{2}(t)\right), \Delta v_{2}(t)\right)\right. \\
& \left.+\rho_{3}(t)\left(\phi_{3}\left(u_{1}(t)\right), v_{1}(t)\right)+\rho_{4}(t)\left(\phi_{4}\left(u_{2}(t)\right), v_{2}(t)\right)\right], \quad \text { for } u, v \in E .
\end{aligned}
$$

It follows from $(\mathcal{A} 2)$ that

$$
\begin{aligned}
\left\langle\mathrm{I}^{\prime}(\mathrm{u})-\mathrm{I}^{\prime}(v), \mathrm{u}-v\right\rangle= & \sum_{\mathrm{t}=1}^{\mathrm{T}}\left[\rho_{1}(\mathrm{t})\left(\phi_{1}\left(\Delta \mathrm{u}_{1}(\mathrm{t})\right)-\phi_{1}\left(\Delta v_{1}(\mathrm{t})\right), \Delta \mathrm{u}_{1}(\mathrm{t})-\Delta v_{1}(\mathrm{t})\right)\right. \\
& +\rho_{2}(\mathrm{t})\left(\phi_{2}\left(\Delta \mathrm{u}_{2}(\mathrm{t})\right)-\phi_{2}\left(\Delta v_{2}(\mathrm{t})\right), \Delta \mathrm{u}_{2}(\mathrm{t})-\Delta v_{2}(\mathrm{t})\right) \\
& +\rho_{3}(\mathrm{t})\left(\phi_{3}\left(\mathrm{u}_{1}(\mathrm{t})\right)-\phi_{3}\left(v_{1}(\mathrm{t})\right), \mathrm{u}_{1}(\mathrm{t})-v_{1}(\mathrm{t})\right) \\
& \left.+\rho_{4}(\mathrm{t})\left(\phi_{4}\left(\mathrm{u}_{1}(\mathrm{t})\right)-\phi_{4}\left(v_{2}(\mathrm{t})\right), \mathrm{u}_{2}(\mathrm{t})-v_{2}(\mathrm{t})\right)\right] \\
\geqslant & \sum_{\mathrm{t}=1}^{\mathrm{T}}\left[\mathrm{c}_{1} \rho_{1}^{-}\left|\Delta \mathrm{u}_{1}(\mathrm{t})-\Delta v_{1}(\mathrm{t})\right|^{\Theta}+\mathrm{c}_{2} \rho_{2}^{-}\left|\Delta \mathrm{u}_{2}(\mathrm{t})-\Delta v_{2}(\mathrm{t})\right|^{\Theta}\right. \\
& \left.+\mathrm{c}_{3} \rho_{3}^{-}\left|\mathrm{u}_{1}(\mathrm{t})-v_{1}(\mathrm{t})\right|^{\Theta}+\mathrm{c}_{4} \rho_{4}^{-}\left|\mathrm{u}_{2}(\mathrm{t})-v_{2}(\mathrm{t})\right|^{\Theta}\right] \\
\geqslant & \min \left\{\mathrm{c}_{1} \rho_{1}^{-}, \mathrm{c}_{3} \rho_{3}^{-}\right\}\left\|\mathrm{u}_{1}-v_{1}\right\|_{\mathrm{E}_{\mathrm{T}}}^{\Theta}+\min \left\{\mathrm{c}_{2} \rho_{2}^{-}, \mathrm{c}_{4} \rho_{4}^{-}\right\}\left\|\mathrm{u}_{2}-v_{2}\right\|_{\mathrm{E}_{\mathrm{T}}}^{\Theta} \\
\geqslant & \frac{1}{2^{\theta-1}} \min \left\{\mathrm{c}_{1} \rho_{1}^{-}, \mathrm{c}_{2} \rho_{2}^{-}, \mathrm{c}_{3} \rho_{3}^{-}, \mathrm{c}_{4} \rho_{4}^{-}\right\}\left(\left\|\mathrm{u}_{1}-v_{1}\right\|_{\mathrm{E}_{\mathrm{T}}}+\left\|\mathrm{u}_{2}-v_{2}\right\|_{\mathrm{E}_{\mathrm{T}}}\right)^{\Theta} \\
= & \frac{1}{2^{\theta-1}} \min \left\{\mathrm{c}_{1} \rho_{1}^{-}, \mathrm{c}_{2} \rho_{2}^{-}, \mathrm{c}_{3} \rho_{3}^{-}, \mathrm{c}_{4} \rho_{4}^{-}\right\}\|\mathrm{u}-v\|^{\Theta}, \quad \text { for } \mathrm{u}, v \in \mathrm{E} .
\end{aligned}
$$

So $\mathrm{I}^{\prime}$ is uniformly monotone in $\mathrm{E}$. By $(\mathcal{A} 1)$ and $(\mathcal{A} 2)$, we have

$$
\left(\phi_{i}(x), x\right) \geqslant c_{i}|x|^{\Theta} \quad \text { for all } x \in \mathbb{R}^{N}, \quad i=1,2,3,4 .
$$

Hence, (3.1) implies that

$$
\begin{aligned}
\frac{\left\langle I^{\prime}(u), u\right\rangle}{\|u\|}= & \frac{1}{\|u\|} \sum_{t=1}^{T}\left[\rho_{1}(t)\left(\phi_{1}\left(\Delta u_{1}(t)\right), \Delta u_{1}(t)\right)+\rho_{2}(t)\left(\phi_{2}\left(\Delta u_{2}(t)\right), \Delta u_{2}(t)\right)\right. \\
& \left.+\rho_{3}(t)\left(\phi_{3}\left(u_{1}(t)\right), u_{1}(t)\right)+\rho_{4}(t)\left(\phi_{4}\left(u_{2}(t)\right), u_{2}(t)\right)\right] \\
\geqslant & \frac{1}{\|u\|}\left\{\sum_{t=1}^{T}\left[c_{1} \rho_{1}^{-}\left|\Delta u_{1}(t)\right|^{\Theta}+c_{2} \rho_{2}^{-}\left|\Delta u_{2}(t)\right|^{\Theta}+c_{3} \rho_{3}^{-}\left|u_{1}(t)\right|^{\theta}+c_{4} \rho_{4}^{-}\left|u_{2}(t)\right|^{\Theta}\right]\right\} \\
\geqslant & \min \left\{c_{1} \rho_{1}^{-}, c_{2} \rho_{2}^{-}, c_{3} \rho_{3}^{-}, c_{4} \rho_{4}^{-}\right\} \frac{\left\|u_{1}\right\|_{E_{T}}^{\Theta}+\left\|u_{2}\right\|_{E_{T}}^{\Theta}}{\left\|u_{1}\right\|_{E_{T}}+\left\|u_{2}\right\|_{E_{T}}}
\end{aligned}
$$




$$
\begin{aligned}
& \geqslant \frac{1}{2^{\theta-1}} \min \left\{c_{1} \rho_{1}^{-}, c_{2} \rho_{2}^{-}, c_{3} \rho_{3}^{-}, c_{4} \rho_{4}^{-}\right\} \frac{\left(\left\|u_{1}\right\|_{E_{\mathrm{T}}}+\left\|u_{2}\right\|_{\mathrm{E}_{\mathrm{T}}}\right)^{\theta}}{\left\|u_{1}\right\|_{\mathrm{E}_{\mathrm{T}}}+\left\|\mathrm{u}_{2}\right\|_{\mathrm{E}_{\mathrm{T}}}} \\
& =\frac{1}{2^{\theta-1}} \min \left\{c_{1} \rho_{1}^{-}, c_{2} \rho_{2}^{-}, c_{3} \rho_{3}^{-}, c_{4} \rho_{4}^{-}\right\}\|u\|^{\theta-1}
\end{aligned}
$$

for all $u \in E$. So $\lim _{\|u\| \rightarrow \infty} \frac{\left\langle I^{\prime}(u), u\right\rangle}{\|u\|}=+\infty$, that is, $I^{\prime}$ is coercive in $E$. Next, we show that $I^{\prime}$ is also hemicontinuous in E. Assume that $s \rightarrow s^{*}, s, s^{*} \in[0,1]$. Note that

$$
\left|\left\langle\mathrm{I}^{\prime}(\mathrm{u}+\mathrm{sv}), w\right\rangle-\left\langle\mathrm{I}^{\prime}\left(\mathrm{u}+\mathrm{s}^{*} v\right), w\right\rangle\right| \leqslant\left\|\mathrm{I}^{\prime}(\mathrm{u}+\mathrm{sv})-\mathrm{I}^{\prime}\left(\mathrm{u}+\mathrm{s}^{*} v\right)\right\|\|w\|
$$

for all $u, v, w \in E$. Then the continuity of $\mathrm{I}^{\prime}$ implies that $\left\langle\mathrm{I}^{\prime}(u+s v), w\right\rangle \rightarrow\left\langle\mathrm{I}^{\prime}\left(u+\mathrm{s}^{*} v\right), w\right\rangle$ as $\mathrm{s} \rightarrow \mathrm{s}^{*}$ for all $u, v, w \in E$. Hence, $\mathrm{I}^{\prime}$ is hemicontinuous in E. Thus by Theorem 26.A in [25], we know that $\mathrm{I}^{\prime}$ admits a continuous inverse in $\mathrm{E}$.

Obviously, $\Psi, \Phi$, and $\Gamma$ are $C^{1}$ functionals. Next, we show that $\Psi^{\prime}, \Phi^{\prime}$, and $\Gamma^{\prime}$ are compact. Assume that $\left\{u^{n}\right\} \subset E$ is bounded. Then there exists a constant $D_{1}$ such that $\left\|u^{n}\right\| \leqslant D_{1}$ and there exists a subsequence, still denoted by $\left\{u_{n}\right\}$, such that $u^{n} \rightarrow u^{*}$ for some $u^{*} \in E$. Furthermore, $u^{n} \rightarrow u^{*}$. By the continuity of $\Psi^{\prime}, \Phi^{\prime}$ and $\Gamma^{\prime}$, it is clear that

$$
\left\|\Psi^{\prime}\left(u^{n}\right)-\Psi^{\prime}\left(u^{*}\right)\right\| \rightarrow 0, \quad\left\|\Phi^{\prime}\left(u^{n}\right)-\Phi^{\prime}\left(u^{*}\right)\right\| \rightarrow 0, \quad\left\|\Gamma^{\prime}\left(u^{n}\right)-\Gamma^{\prime}\left(u^{*}\right)\right\| \rightarrow 0, \quad \text { as } n \rightarrow \infty .
$$

Hence, $\Psi^{\prime}, \Phi^{\prime}$, and $\Gamma^{\prime}$ are compact in $\mathrm{E}$. It follows from $(\mathcal{A} 4)$ that

$$
\Psi(u)+\lambda \Phi(u)=\sum_{t=1}^{T}\left[\lambda G\left(t, u_{1}(t), u_{2}(t)\right)-F\left(t, u_{1}(t), u_{2}(t)\right)\right] \geqslant T C_{0}(\lambda),
$$

which shows that $\Psi+\lambda \Phi$ is bounded below for all $\lambda>0$. Moreover, $(\mathcal{A} 4)$ implies that for any positive constant $D_{1}$, there exists a positive constant $D_{2}\left(D_{1}\right)$, which depends on $D_{1}$, such that

$$
\mathrm{F}\left(\mathrm{t}, \mathrm{x}_{1}, \mathrm{x}_{2}\right) \geqslant \mathrm{D}_{1}\left(\left|\mathrm{x}_{1}\right|^{\mathrm{l}}+\left|\mathrm{x}_{2}\right|^{\mathrm{l}}\right)-\mathrm{D}_{2}\left(\mathrm{D}_{1}\right)
$$

for all $\left(x_{1}, x_{2}\right) \in \mathbb{R}^{N} \times \mathbb{R}^{N}$ and $t \in \mathbb{Z}[1, T]$. Then (3.2), (A3), (2.2), and (2.3) imply that

$$
\begin{aligned}
\lim _{\|u\| \rightarrow \infty} \frac{\Psi(u)}{I(u)}= & \lim _{\|u\| \rightarrow \infty} \frac{-\sum_{t=1}^{T} F\left(t, u_{1}(t), u_{2}(t)\right)}{\sum_{t=1}^{T}\left[\rho_{1}(t) \Phi_{1}\left(\Delta u_{1}(t)\right)+\rho_{2}(t) \Phi_{2}\left(\Delta u_{2}(t)\right)+\rho_{3}(t) \Phi_{3}\left(u_{1}(t)\right)+\rho_{4}(t) \Phi_{4}\left(u_{2}(t)\right)\right]} \\
\leqslant & \lim _{\|u\| \rightarrow \infty} \frac{-D_{1} \sum_{t=1}^{T}\left(\left|u_{1}(t)\right|^{l}+\left|u_{2}(t)\right|^{l}\right)+D_{2}\left(D_{1}\right) T}{\sum_{t=1}^{T}\left[\rho_{1}(t) \Phi_{1}\left(\Delta u_{1}(t)\right)+\rho_{2}(t) \Phi_{2}\left(\Delta u_{2}(t)\right)+\rho_{3}(t) \Phi_{3}\left(u_{1}(t)\right)+\rho_{4}(t) \Phi_{4}\left(u_{2}(t)\right)\right]} \\
\leqslant & \lim _{\|u\| \rightarrow \infty} \frac{-D_{1} \sum_{t=1}^{T}\left(\left|u_{1}(t)\right|^{l}+\left|u_{2}(t)\right|^{l}\right)}{\sum_{t=1}^{T}\left[d_{1} \rho_{1}^{+}\left|\Delta u_{1}(t)\right|^{l}+d_{2} \rho_{2}^{+}\left|\Delta u_{2}(t)\right|^{l}+d_{3} \rho_{3}^{+}\left|u_{1}(t)\right|^{l}+d_{4} \rho_{4}^{+}\left|u_{2}(t)\right|^{l}+\sum_{i=1}^{4} m_{i} \rho_{i}^{+}\right]} \\
& +\lim _{\|u\| \rightarrow \infty} \frac{D_{2}\left(D_{1}\right) T}{\min \left\{\rho_{1}^{-}, \rho_{2}^{-}, \rho_{2}^{-}, \rho_{4}^{-}\right\} \sum_{t=1}^{T}\left[\Phi_{1}\left(\Delta u_{1}(t)\right)+\Phi_{2}\left(\Delta u_{2}(t)\right)+\Phi_{3}\left(u_{1}(t)\right)+\Phi_{4}\left(u_{2}(t)\right)\right]} \\
\leqslant & \lim _{\|u\| \rightarrow \infty} \frac{-D_{1} C_{3}^{l}\left(\left\|u_{1}\right\|_{E_{T}}^{l}+\left\|u_{2}\right\|_{E_{T}}^{l}\right)}{\max \left\{d_{1} \rho_{1}^{+}, d_{2} \rho_{2}^{+}, d_{3} \rho_{3}^{+}, d_{4} \rho_{4}^{+}\right\}\left(\left\|u_{1}\right\|_{\left[E_{T}\right]}^{l}+\left\|u_{2}\right\|_{\left[E_{T}\right]}^{l}\right)+\sum_{t=1}^{T} \sum_{i=1}^{4} m_{i} \rho_{i}^{+}}
\end{aligned}
$$




$$
\begin{aligned}
& \leqslant \lim _{\|u\| \rightarrow \infty} \frac{-D_{1} c_{3 \frac{1}{2^{l-1}}}^{l}\left(\left\|u_{1}\right\|_{E_{\mathrm{T}}}+\left\|u_{2}\right\|_{E_{\mathrm{T}}}\right)^{l}}{\max \left\{d_{1} \rho_{1}^{+}, d_{2} \rho_{2}^{+}, d_{3} \rho_{3}^{+}, d_{4} \rho_{4}^{+}\right\}\left(\left\|u_{1}\right\|_{\left[E_{T}\right]}^{l}+\left\|u_{2}\right\|_{\left[E_{T}\right]}^{l}\right)+\sum_{t=1}^{T} \sum_{i=1}^{4} m_{i} \rho_{i}^{+}} \\
& \leqslant \lim _{\|\mathfrak{u}\| \rightarrow \infty} \frac{-\mathrm{D}_{1} \mathrm{C}_{3}^{\mathrm{l}} \frac{1}{2^{\mathrm{l}-1}}\left(\left\|\mathrm{u}_{1}\right\|_{\mathrm{E}_{\mathrm{T}}}+\left\|\mathrm{u}_{2}\right\|_{\mathrm{E}_{\mathrm{T}}}\right)^{\mathrm{l}}}{\max \left\{\mathrm{d}_{1} \rho_{1}^{+}, \mathrm{d}_{2} \rho_{2}^{+}, \mathrm{d}_{3} \rho_{3}^{+}, \mathrm{d}_{4} \rho_{4}^{+}\right\}\left(\mathrm{C}_{6}^{\mathrm{l}}\left\|\mathrm{u}_{1}\right\|_{\mathrm{E}_{\mathrm{T}}}^{\mathrm{l}}+\mathrm{C}_{6}^{\mathrm{l}}\left\|\mathrm{u}_{2}\right\|_{\mathrm{E}_{\mathrm{T}}}^{\mathrm{l}}\right)+\sum_{\mathrm{t}=1}^{\mathrm{T}} \sum_{\mathrm{i}=1}^{4} \mathrm{~m}_{\mathrm{i}} \rho_{\mathrm{i}}^{+}} \\
& \leqslant \lim _{\|u\| \rightarrow \infty} \frac{-D_{1} C_{3}^{l} \frac{1}{2^{l-1}}\left(\left\|u_{1}\right\|_{E_{\mathrm{T}}}+\left\|u_{2}\right\|_{E_{\mathrm{T}}}\right)^{\mathrm{l}}}{\max \left\{d_{1} \rho_{1}^{+}, d_{2} \rho_{2}^{+}, d_{3} \rho_{3}^{+}, d_{4} \rho_{4}^{+}\right\} C_{6}^{l}\left(\left\|u_{1}\right\|_{E_{\mathrm{T}}}+\left\|u_{2}\right\|_{E_{\mathrm{T}}}\right)^{l}+\sum_{t=1}^{T} \sum_{i=1}^{4} m_{i} \rho_{i}^{+}} \\
& =\frac{1}{2^{l-1}} \frac{-D_{1} C_{3}^{l}}{\max \left\{d_{1} \rho_{1}^{+}, d_{2} \rho_{2}^{+}, d_{3} \rho_{3}^{+}, d_{4} \rho_{4}^{+}\right\} C_{6}^{l}} .
\end{aligned}
$$

By arbitrariness of $\mathrm{D}_{1}$, we obtain that

$$
\lim _{\|u\| \rightarrow \infty} \frac{\Psi(u)}{I(u)}=-\infty
$$

By $(\mathcal{A} 1)$ and $(\mathcal{A} 3)$, we know that $\Phi_{i}$ reaches its unique minimum at $0, i=1,2,3,4$ (see [11]) and so I has unique global minima 0 . Then $M=\{0\}$. By $(\mathcal{A} 5)$, we have $\sup _{M} \Phi=0$. Hence, by Theorem 2.3 , the conclusion of Theorem 1.2 holds.

Proof of Corollary 1.3. It follows from $(\mathcal{A} 4)^{\prime}$ that there exist $\mathrm{D}_{3}>0$ and $\mathrm{D}_{4}>0$ such that for every $\mathrm{t} \in$ $\mathbb{Z}[1, T]$,

$$
\mathrm{F}\left(\mathrm{t}, \mathrm{x}_{1}, \mathrm{x}_{2}\right) \leqslant \mathrm{D}_{3}\left|\mathrm{x}_{1}\right|^{\mathrm{s}}+\mathrm{D}_{3}\left|\mathrm{x}_{2}\right|^{\mathrm{s}}+\mathrm{D}_{4}
$$

and for any $D_{5}>D_{3}$, there is a constant $D_{6}\left(D_{5}\right)$, which depends on $D_{5}$, such that

$$
G\left(t, x_{1}, x_{2}\right) \geqslant D_{5}\left|x_{1}\right|^{s}+D_{5}\left|x_{2}\right|^{s}+D_{6}\left(D_{5}\right) .
$$

Obviously, for every $\lambda>0$, we can find a sufficiently large $D_{5}(\lambda)$ such that $\lambda D_{5}(\lambda)>D_{3}$. Hence, we have

$$
\lambda G\left(t, x_{1}, x_{2}\right) \geqslant D_{3}\left|x_{1}\right|^{s}+D_{3}\left|x_{2}\right|^{s}+\lambda D_{6}\left(D_{5}(\lambda)\right) \geqslant F\left(t, x_{1}, x_{2}\right)-D_{4}+\lambda D_{6}\left(D_{5}(\lambda)\right) .
$$

So $(\mathcal{A} 4)^{\prime}$ implies $(\mathcal{A} 4)$.

\section{Proof of Theorem 1.4}

When the condition $(\gamma)$ holds, on $E_{T}$, we define

$$
\|u\|_{\left(E_{T, q}\right)}=\left(\sum_{t=1}^{T} \gamma_{1}(t)\left|\Delta u_{1}(t)\right|^{q}+\sum_{t=1}^{T} \gamma_{3}(t)\left|u_{1}(t)\right|^{q}\right)^{1 / q}
$$

and

$$
\|u\|_{\left(E_{T, p}\right)}=\left(\sum_{t=1}^{T} \gamma_{2}(t)\left|\Delta u_{2}(t)\right|^{p}+\sum_{t=1}^{T} \gamma_{4}(t)\left|u_{2}(t)\right|^{p}\right)^{1 / p}
$$

For $u=\left(u_{1}, u_{2}\right) \in E$, define

$$
\|u\|_{(\infty)}=\left\|u_{1}\right\|_{\infty}+\left\|u_{2}\right\|_{\infty} .
$$

Moreover, it is clear that $E$ is homeomorphic to $\mathbb{R}^{2 N T}$. Then there is a basis of $E$ denoted by $\left\{e_{1}, e_{2}, \ldots, e_{2 N T}\right\}$. For every $u \in E$, there exists a unique point $\left(\lambda_{1}, \lambda_{2}, \ldots, \lambda_{2 N T}\right) \in \mathbb{R}^{2 N T}$ such that

$$
u=\sum_{i=1}^{2 N T} \lambda_{i} e_{i}
$$


and define

$$
\|u\|_{(2)}=\left(\sum_{i=1}^{2 N T} \lambda_{i}^{2}\right)^{\frac{1}{2}}
$$

Set

$$
E_{\delta}=\left\{u \in E:\|u\|_{(2)}=\delta\right\} .
$$

Since both $E$ and $E_{T}$ are finite-dimensional spaces, then $\|\cdot\|_{(\infty)}$ is equivalent to $\|\cdot\|_{(2)}$ on $E$, and both $\|\cdot\|_{\left(E_{T}, q\right)}$ and $\|\cdot\|_{\left(E_{T}, p\right)}$ are equivalent to $\|\cdot\|_{\infty}$ on $E_{T}$. Hence, there exist positive constants $R_{i}(i=$ $1,2, \cdots, 6)$ such that

$$
\begin{aligned}
& R_{1}\|\cdot\|_{(2)} \leqslant\|\cdot\|_{(\infty)} \leqslant R_{2}\|\cdot\|_{(2)}, \\
& R_{3}\|\cdot\|_{\infty} \leqslant\|\cdot\|_{\left(E_{T, q}\right)} \leqslant R_{4}\|\cdot\|_{\infty}, \\
& R_{5}\|\cdot\|_{\infty} \leqslant\|\cdot\|_{\left(E_{T, p}\right)} \leqslant R_{6}\|\cdot\|_{\infty} .
\end{aligned}
$$

In Lemma 2.1, let

$$
\mathrm{L}\left(\mathrm{t}, \mathrm{x}_{1}, \mathrm{x}_{2}, \mathrm{y}_{1}, \mathrm{y}_{2}\right)=\gamma_{1}(\mathrm{t}) \Phi_{1}\left(\mathrm{y}_{1}\right)+\gamma_{2}(\mathrm{t}) \Phi_{2}\left(\mathrm{y}_{2}\right)+\gamma_{3}(\mathrm{t}) \Phi_{3}\left(\mathrm{x}_{1}\right)+\gamma_{4}(\mathrm{t}) \Phi_{4}\left(\mathrm{x}_{2}\right)-\mathrm{F}\left(\mathrm{t}, \mathrm{x}_{1}, \mathrm{x}_{2}\right),
$$

where $\mathrm{F}: \mathbb{Z}[1, T] \times \mathbb{R}^{\mathrm{N}} \times \mathbb{R}^{\mathrm{N}} \longrightarrow \mathbb{R}$ is continuously differentiable in $\left(x_{1}, x_{2}\right) \in \mathbb{R}^{\mathrm{N}} \times \mathbb{R}^{\mathrm{N}}$ for all $\mathrm{t} \in \mathbb{Z}[1, \mathrm{~T}]$. Then

$$
\begin{aligned}
\varphi(u)= & \sum_{t=1}^{T} \gamma_{1}(t) \Phi_{1}\left(\Delta u_{1}(t)\right)+\sum_{t=1}^{T} \gamma_{2}(t) \Phi_{2}\left(\Delta u_{2}(t)\right) \\
& +\sum_{t=1}^{T} \gamma_{3}(t) \Phi_{3}\left(u_{1}(t)\right)+\sum_{t=1}^{T} \gamma_{4}(t) \Phi_{4}\left(u_{2}(t)\right)-\sum_{t=1}^{T} F\left(t, u_{1}(t), u_{2}(t)\right) .
\end{aligned}
$$

And for all $u, v \in E$, we have

$$
\begin{aligned}
\left\langle\varphi^{\prime}(\mathrm{u}), v\right\rangle= & \left\langle\varphi^{\prime}\left(\mathrm{u}_{1}, \mathrm{u}_{2}\right),\left(v_{1}, v_{2}\right)\right\rangle \\
= & \sum_{\mathrm{t}=1}^{\mathrm{T}}\left[\gamma_{1}(\mathrm{t})\left(\phi_{1}\left(\Delta \mathrm{u}_{1}(\mathrm{t})\right), \Delta v_{1}(\mathrm{t})\right)+\gamma_{2}(\mathrm{t})\left(\phi_{2}\left(\Delta \mathrm{u}_{2}(\mathrm{t})\right), \Delta v_{2}(\mathrm{t})\right)\right. \\
& \left.+\gamma_{3}(\mathrm{t})\left(\phi_{3}\left(\mathrm{u}_{1}(\mathrm{t})\right), v_{1}(\mathrm{t})\right)+\gamma_{4}(\mathrm{t})\left(\phi_{4}\left(\mathrm{u}_{2}(\mathrm{t})\right), v_{2}(\mathrm{t})\right)\right] \\
& -\sum_{\mathrm{t}=1}^{\mathrm{T}}\left(\nabla_{\mathrm{u}_{1}} \mathrm{~F}\left(\mathrm{t}, \mathrm{u}_{1}(\mathrm{t}), \mathrm{u}_{2}(\mathrm{t})\right), v_{1}(\mathrm{t})\right)-\sum_{\mathrm{t}=1}^{\mathrm{T}}\left(\nabla_{\mathrm{u}_{2}} \mathrm{~F}\left(\mathrm{t}, \mathrm{u}_{1}(\mathrm{t}), \mathrm{u}_{2}(\mathrm{t})\right), v_{2}(\mathrm{t})\right) .
\end{aligned}
$$

Similar to the argument of Lemma 2.2, it is easy to obtain the following lemma.

Lemma 4.1. If $u \in E$ is a solution of Euler equation $\varphi^{\prime}(u)=0$, then $u$ is a solution of system (1.5).

Denote with $\theta$ the zero element of $X$ and with $\Sigma$ the family of sets $A \subset X \backslash\{\theta\}$ such that $A$ is closed in $X$ and symmetric with respect to $\theta$, i.e. $u \in A$ implies $-u \in A$.

Theorem 4.2 ([16, Theorem 9.1]). Let $X$ be a real Banach space and $\varphi$ be an even function belonging to $C^{1}(X, \mathbb{R})$ with $\varphi(\theta)=0$, bounded from below and satisfying (PS) condition. Suppose that there is a set $\mathrm{K} \in \Sigma$ such that $\mathrm{K}$ is homeomorphic to $\mathrm{S}^{\mathrm{j}-1}\left(\mathrm{j}-1\right.$ dimension unit sphere) by an odd map and $\sup _{\mathrm{K}} \varphi<0$. Then, $\varphi$ has at least $\mathrm{j}$ distinct pairs of nonzero critical points. 
Proof of Theorem 1.4. It follows from $(\phi),(4.4),(2.1),(4.2),(4.3)$, and (F0) that

$$
\begin{aligned}
\varphi(u)= & \sum_{t=1}^{T} \gamma_{1}(t) \Phi_{1}\left(\Delta u_{1}(t)\right)+\sum_{t=1}^{T} \gamma_{2}(t) \Phi_{2}\left(\Delta u_{2}(t)\right) \\
& +\sum_{t=1}^{T} \gamma_{3}(t) \Phi_{3}\left(u_{1}(t)\right)+\sum_{t=1}^{T} \gamma_{4}(t) \Phi_{4}\left(u_{2}(t)\right)-\sum_{t=1}^{T} F\left(t, u_{1}(t), u_{2}(t)\right) \\
\geqslant & a_{1} \sum_{t=1}^{T} \gamma_{1}(t)\left|\Delta u_{1}(t)\right|^{q}+a_{2} \sum_{t=1}^{T} \gamma_{2}(t)\left|\Delta u_{2}(t)\right|^{p} \\
& +a_{3} \sum_{t=1}^{T} \gamma_{3}(t)\left|u_{1}(t)\right|^{q}+a_{4} \sum_{t=1}^{T} \gamma_{4}(t)\left|u_{2}(t)\right|^{p}-\sum_{t=1}^{T} F\left(t, u_{1}(t), u_{2}(t)\right) \\
\geqslant & \min \left\{a_{1}, a_{3}\right\}\left\|u_{1}\right\|_{\left(E_{T, q}\right)}^{q}+\min \left\{a_{2}, a_{4}\right\}\left\|u_{2}\right\|_{\left(E_{T, p}\right)}^{p}-\sum_{t=1}^{T}\left[h_{1}(t)\left|u_{1}(t)\right|^{\alpha_{1}}+h_{2}(t)\left|u_{2}(t)\right|^{\alpha_{2}}+l(t)\right] \\
\geqslant & \min \left\{a_{1}, a_{3}\right\} R_{3}^{q}\left\|u_{1}\right\|_{\infty}^{q}+\min \left\{a_{2}, a_{4}\right\} R_{5}^{p}\left\|u_{2}\right\|_{\infty}^{p}-\left\|u_{1}\right\|_{\infty}^{\alpha_{1}} \sum_{t=1}^{T} h_{1}(t)-\left\|u_{2}\right\|_{\infty}^{\alpha_{2}} \sum_{t=1}^{T} h_{2}(t)-\sum_{t=1}^{T} l(t)
\end{aligned}
$$

for all $u \in E$. Since $\alpha_{1} \in[0, q)$ and $\alpha_{2} \in[0, p)$, it is easy to see that

$$
\varphi(u) \rightarrow+\infty, \text { as }\|u\|_{(\infty)}=\left\|u_{1}\right\|_{\infty}+\left\|u_{2}\right\|_{\infty} \rightarrow \infty,
$$

which implies that $\varphi$ is bounded from below and any (PS) sequence $\left\{u_{n}\right\}$ is bounded. Hence $\varphi$ satisfies (PS) condition. Obviously, (F1) and (F2) imply that $\varphi(0)=0$ and $\varphi$ is even. Next, we prove that there exists a set $K \subset E$ such that $K$ is homeomorphic to $S^{2 N T-1}$ by an odd map, and $\sup _{K} \varphi<0$. Note that $\delta<1$. For all $u=\left(u_{1}, u_{2}\right) \in E_{\delta}$ and $r>0$, by (4.1) we have

$$
\begin{aligned}
M_{1} r^{\beta_{1}} & \left\|u_{1}\right\|_{\infty}^{\beta_{1}}+M_{2} r^{\beta_{2}}\left\|u_{2}\right\|_{\infty}^{\beta_{2}} \\
& =M_{1} r^{\beta_{1}} R_{2}^{\beta_{1}}\left\|\frac{u_{1}}{R_{2}}\right\|_{\infty}^{\beta_{1}}+M_{2} r^{\beta_{2}} R_{2}^{\beta_{2}}\left\|\frac{u_{2}}{R_{2}}\right\|_{\infty}^{\beta_{2}} \\
& \geqslant \min \left\{M_{1} r^{\beta_{1}} R_{2}^{\beta_{1}}, M_{2} r^{\beta_{2}} R_{2}^{\beta_{2}}\right\}\left(\left\|\frac{u_{1}}{R_{2}}\right\|_{\infty}^{\max \left\{\beta_{1}, \beta_{2}\right\}}+\left\|\frac{u_{2}}{R_{2}}\right\|_{\infty}^{\max \left\{\beta_{1}, \beta_{2}\right\}}\right) \\
& \geqslant 2^{1-\max \left\{\beta_{1}, \beta_{2}\right\}} \min \left\{M_{1} r^{\beta_{1}} R_{2}^{\beta_{1}}, M_{2} r^{\beta_{2}} R_{2}^{\beta_{2}}\right\}\left(\left\|\frac{u_{1}}{R_{2}}\right\|_{\infty}+\left\|\frac{u_{2}}{R_{2}}\right\|_{\infty}\right)^{\max \left\{\beta_{1}, \beta_{2}\right\}} \\
& =2^{1-\max \left\{\beta_{1}, \beta_{2}\right\}} \min \left\{M_{1} r^{\beta_{1}} R_{2}^{\beta_{1}}, M_{2} r^{\beta_{2}} R_{2}^{\beta_{2}}\right\}\left(\frac{1}{R_{2}}\right)^{\max \left\{\beta_{1}, \beta_{2}\right\}}\|u\|_{(\infty)}^{\max \left\{\beta_{1}, \beta_{2}\right\}} \\
& \geqslant 2^{1-\max \left\{\beta_{1}, \beta_{2}\right\}} \min \left\{M_{1} r^{\beta_{1}} R_{2}^{\beta_{1}}, M_{2} r^{\beta_{2}} R_{2}^{\beta_{2}}\right\}\left(\frac{1}{R_{2}}\right)^{\max \left\{\beta_{1}, \beta_{2}\right\}} R_{1}^{\max \left\{\beta_{1}, \beta_{2}\right\}}\|u\|_{(2)}^{\max \left\{\beta_{1}, \beta_{2}\right\}} \\
& =2 \min \left\{M_{1} r^{\beta_{1}} R_{2}^{\beta_{1}}, M_{2} r^{\beta_{2}} R_{2}^{\beta_{2}}\right\}\left(\frac{R_{1} \delta}{2 R_{2}}\right)^{\max \left\{\beta_{1}, \beta_{2}\right\}} .
\end{aligned}
$$

Then for all $u=\left(u_{1}, u_{2}\right) \in E_{\delta}$ and $0<r<\frac{1}{R_{2}}$, by $(\phi),(\mathcal{F} 3),(2.1),(4.1)-(4.3)$, and (4.5) we have

$$
\begin{aligned}
\varphi(r u)= & \sum_{\mathfrak{t}=1}^{T} \gamma_{1}(t) \Phi_{1}\left(r \Delta u_{1}(t)\right)+\sum_{t=1}^{T} \gamma_{2}(t) \Phi_{2}\left(r \Delta u_{2}(t)\right) \\
& +\sum_{t=1}^{T} \gamma_{3}(t) \Phi_{3}\left(r u_{1}(t)\right)+\sum_{t=1}^{T} \gamma_{4}(t) \Phi_{4}\left(r u_{2}(t)\right)-\sum_{t=1}^{T} F\left(t, r u_{1}(t), r u_{2}(t)\right)
\end{aligned}
$$




$$
\begin{aligned}
& \leqslant b_{1} \sum_{t=1}^{T} \gamma_{1}(t)\left|r \Delta u_{1}(t)\right|^{q}+b_{2} \sum_{t=1}^{T} \gamma_{2}(t)\left|r \Delta u_{2}(t)\right|^{p} \\
& +b_{3} \sum_{t=1}^{T} \gamma_{3}(t)\left|r u_{1}(t)\right|^{q}+b_{4} \sum_{t=1}^{T} \gamma_{4}(t)\left|r u_{2}(t)\right|^{p}-\sum_{t=1}^{T} F\left(t, r u_{1}(t), r u_{2}(t)\right) \\
& \leqslant \max \left\{b_{1}, b_{3}\right\} r^{q}\left\|u_{1}\right\|_{\left(E_{T}, q\right)}^{q}+\max \left\{b_{2}, b_{4}\right\} r^{p}\left\|u_{2}\right\|_{\left(E_{T}, p\right)}^{p} \\
& -M_{1} r^{\beta_{1}} \sum_{t=1}^{T}\left|u_{1}(t)\right|^{\beta_{1}}-M_{2} r^{\beta_{2}} \sum_{t=1}^{T}\left|u_{2}(t)\right|^{\beta_{2}} \\
& \leqslant \max \left\{b_{1}, b_{3}\right\} r^{q} R_{4}^{q}\left\|u_{1}\right\|_{\infty}^{q}+\max \left\{b_{2}, b_{4}\right\} r^{p} R_{6}^{p}\left\|u_{2}\right\|_{\infty}^{p}-M_{1} r^{\beta_{1}}\left\|u_{1}\right\|_{\infty}^{\beta_{1}}-M_{2} r^{\beta_{2}}\left\|u_{2}\right\|_{\infty}^{\beta_{2}} \\
& \leqslant \max \left\{b_{1}, b_{3}\right\} r^{q} R_{4}^{q} R_{2}^{q}\|u\|_{(2)}^{q}+\max \left\{b_{2}, b_{4}\right\} r^{p} R_{6}^{p} R_{2}^{p}\|u\|_{(2)}^{p} \\
& -2 \min \left\{M_{1} r^{\beta_{1}} R_{2}^{\beta_{1}}, M_{2} r^{\beta_{2}} R_{2}^{\beta_{2}}\right\}\left(\frac{R_{1} \delta}{2 R_{2}}\right)^{\max \left\{\beta_{1}, \beta_{2}\right\}} \\
& =\max \left\{b_{1}, b_{3}\right\} r^{q}\left(R_{4} R_{2} \delta\right)^{q}+\max \left\{b_{2}, b_{4}\right\} r^{p}\left(R_{6} R_{2} \delta\right)^{p} \\
& -2 \min \left\{M_{1} r^{\beta_{1}} R_{2}^{\beta_{1}}, M_{2} r^{\beta_{2}} R_{2}^{\beta_{2}}\right\}\left(\frac{R_{1} \delta}{2 R_{2}}\right)^{\max \left\{\beta_{1}, \beta_{2}\right\}} \text {. }
\end{aligned}
$$

Since $\beta_{i} \in(1, \min \{q, p\}), i=1,2$, then (4.6) implies that there exist sufficiently small $r_{0} \in(0,1)$ and $\epsilon>0$ such that $\varphi\left(r_{0} u\right)<-\epsilon$ for all $u \in E_{\delta}$. Set

$$
E_{\delta}^{r_{0}}=\left\{r_{0} u: u \in E_{\delta}\right\} \quad \text { and } \quad s^{2 N T-1}=\left\{\left(\lambda_{1}, \lambda_{2}, \cdots, \lambda_{2 N T}\right) \in \mathbb{R}^{2 N T}: \sum_{i=1}^{2 N T} \lambda_{i}^{2}=1\right\} .
$$

Then $E_{\delta}^{r_{0}} \in \Sigma$ and

$$
\psi(u)<-\epsilon, \quad \forall u \in E_{\delta}^{r_{0}} .
$$

Define the map $\psi: \mathrm{E}_{\delta}^{\mathrm{r}_{0}} \rightarrow \mathrm{S}^{2 \mathrm{NT}-1}$ by

$$
\psi(u)=\psi\left(\sum_{i=1}^{2 N T} \lambda_{i} e_{i}\right)=\frac{1}{r_{0} \delta}\left(\lambda_{1}, \lambda_{2}, \cdots, \lambda_{2 N T}\right) .
$$

Then it is easy to see that $\psi$ is an odd and homeomorphic map. Moreover, (4.7) implies that $\sup _{E_{\delta}^{r_{0}}} \varphi \leqslant$ $-\epsilon<0$. Therefore, by Theorem 4.2, we obtain that system (1.5) has at least 2NT distinct pairs of solutions in $\mathrm{E}$.

\section{Examples}

Example 5.1. We present an example to which Theorem 1.2 is applied and make an estimate for the parameters in our result. Let $T=2$ and $N$ be fixed integer. Assume that $\phi_{1}(y)=y+|y|^{\frac{1}{3}} y, \phi_{2}(y)=$ $y+|y|^{\frac{1}{2}} y, \phi_{3}(y)=\phi_{4}(y)=2 y, \rho_{i}$ are 2-periodic and satisfy $\rho_{i}>0$ for all $t \in \mathbb{Z}[1,2](i=1,2,3,4)$. Then $\Phi_{1}(y)=\frac{|y|^{2}}{2}+\frac{|y|^{\frac{7}{3}}}{\frac{7}{3}}, \Phi_{2}(y)=\frac{|y|^{2}}{2}+\frac{|y|^{\frac{5}{2}}}{\frac{5}{2}}, \Phi_{3}(y)=\Phi_{4}(y)=|y|^{2}$. Let

$$
\begin{aligned}
\mathrm{F}\left(\mathrm{t}, \mathrm{x}_{1}, \mathrm{x}_{2}\right) & =\left|\mathrm{x}_{1}\right|^{3}+\left|\mathrm{x}_{2}\right|^{3}, \\
\mathrm{G}\left(\mathrm{t}, \mathrm{x}_{1}, \mathrm{x}_{2}\right) & =\left|\mathrm{x}_{1}\right|^{4}+\left|\mathrm{x}_{2}\right|^{4}, \\
\mathrm{H}\left(\mathrm{t}, \mathrm{x}_{1}, \mathrm{x}_{2}\right) & =\left(\cos ^{2} \frac{\pi \mathrm{t}}{2}+2\right) \sin \left(\left|\mathrm{x}_{1}\right|^{2}+\left|\mathrm{x}_{2}\right|^{2}+2\right), \\
\mathrm{W}\left(\mathrm{t}, \mathrm{x}_{1}, \mathrm{x}_{2}\right) & =\mathrm{F}\left(\mathrm{t}, \mathrm{x}_{1}, \mathrm{x}_{2}\right)-\lambda \mathrm{G}\left(\mathrm{t}, \mathrm{x}_{1}, \mathrm{x}_{2}\right)+v \mathrm{H}\left(\mathrm{t}, \mathrm{x}_{1}, \mathrm{x}_{2}\right) .
\end{aligned}
$$


Then

$$
\begin{aligned}
I(u)= & \sum_{t=1}^{2}\left[\rho_{1}(t)\left(\frac{\left|\Delta u_{1}(t)\right|^{2}}{2}+\frac{\left|\Delta u_{1}(t)\right|^{\frac{7}{3}}}{\frac{7}{3}}\right)+\rho_{2}(t)\left(\frac{\left|\Delta u_{2}(t)\right|^{2}}{2}+\frac{\left|\Delta u_{2}(t)\right|^{\frac{5}{2}}}{\frac{5}{2}}\right)\right] \\
& +\sum_{t=1}^{2}\left[\rho_{3}(t)\left|u_{1}(t)\right|^{2}+\rho_{4}(t)\left|u_{2}(t)\right|^{2}\right], \\
\Psi(u)= & -\sum_{t=1}^{2}\left(\left|u_{1}(t)\right|^{3}+\left|u_{2}(t)\right|^{3}\right), \\
\Phi(u)= & \sum_{t=1}^{2}\left(\left|u_{1}(t)\right|^{4}+\left|u_{2}(t)\right|^{4}\right), \\
\Gamma(u)= & -\sum_{t=1}^{T}\left(\cos ^{2} \frac{\pi t}{2}+2\right) \sin \left(\left|u_{1}(t)\right|^{2}+\left|u_{2}(t)\right|^{2}+2\right), \quad u \in E .
\end{aligned}
$$

Take $\theta=2$ and $l=\frac{5}{2}$. Hence,

$$
\begin{aligned}
\left(\phi_{1}(x)-\phi_{1}(y), x-y\right)=\left(x+|x|^{\frac{1}{3}} x-y-|y|^{\frac{1}{3}} y, x-y\right) & =(x-y, x-y)+\left(|x|^{\frac{1}{3}} x-|y|^{\frac{1}{3}} y, x-y\right) \\
& \geqslant|x-y|^{2}, \quad \forall x, y \in \mathbb{R}^{N} .
\end{aligned}
$$

Similarly, we have

$$
\left(\phi_{2}(x)-\phi_{2}(y), x-y\right) \geqslant|x-y|^{2}, \quad \forall x, y \in \mathbb{R}^{N} .
$$

So $\phi_{1}$ and $\phi_{2}$ satisfy $(\mathcal{A} 2)$. Moreover, it is obvious that $\phi_{3}$ and $\phi_{4}$ also satisfy $(\mathcal{A} 2)$.

It is easy to see that $\mathrm{F}, \mathrm{G} \in \mathcal{C},(\mathcal{A} 5)$ holds and $\Phi_{i}$ satisfy $(\mathcal{A} 1)$ and $(\mathcal{A} 3), i=1,2,3,4$. Moreover,

$$
\lim _{\left|x_{1}\right|+\left|x_{2}\right| \rightarrow \infty} \frac{F\left(t, x_{1}, x_{2}\right)}{\left|x_{1}\right|^{l}+\left|x_{2}\right|^{l}}=\lim _{\left|x_{1}\right|+\left|x_{2}\right| \rightarrow \infty} \frac{\left|x_{1}\right|^{3}+\left|x_{2}\right|^{3}}{\left|x_{1}\right|^{\frac{5}{2}}+\left|x_{2}\right|^{\frac{5}{2}}}=+\infty
$$

and for all $\lambda>0$,

$$
\lim _{\left|x_{1}\right|+\left|x_{2}\right| \rightarrow \infty} \frac{\lambda G\left(t, x_{1}, x_{2}\right)}{F\left(t, x_{1}, x_{2}\right)}=\lim _{\left|x_{1}\right|+\left|x_{2}\right| \rightarrow \infty} \frac{\lambda\left(\left|x_{1}\right|^{4}+\left|x_{2}\right|^{4}\right)}{\left|x_{1}\right|^{3}+\left|x_{2}\right|^{3}}=+\infty .
$$

Hence, $(\mathcal{A} 4)$ holds. By a simply computation, we have

$$
\gamma=\inf _{E}(\Psi(u)+\Phi(u))=\inf _{E} \sum_{t=1}^{2}\left(\left|u_{1}(t)\right|^{4}+\left|u_{2}(t)\right|^{4}-\left|u_{1}(t)\right|^{3}-\left|u_{2}(t)\right|^{3}\right)=-\frac{27}{64},
$$

which is obtained when $\left|\mathfrak{u}_{1}(1)\right|=\left|\mathfrak{u}_{1}(2)\right|=\left|\mathfrak{u}_{2}(1)\right|=\left|\mathfrak{u}_{2}(2)\right|=\frac{3}{4}$. Moreover, for $r>0$, we have

$$
\begin{aligned}
\Phi^{-1}(r) & =\left\{u \in E:\left|u_{1}(1)\right|^{4}+\left|u_{1}(2)\right|^{4}+\left|u_{2}(1)\right|^{4}+\left|u_{2}(2)\right|^{4}=r\right\}, \\
\left.\left.\Phi^{-1}(]-\infty, r\right]\right) & =\left\{u \in E:\left|u_{1}(1)\right|^{4}+\left|u_{1}(2)\right|^{4}+\left|u_{2}(1)\right|^{4}+\left|u_{2}(2)\right|^{4} \leqslant r\right\}, \\
\Phi^{-1}(] r,+\infty[) & =\left\{u \in E:\left|u_{1}(1)\right|^{4}+\left|u_{1}(2)\right|^{4}+\left|u_{2}(1)\right|^{4}+\left|u_{2}(2)\right|^{4}>r\right\} .
\end{aligned}
$$

Then

$$
\begin{aligned}
\eta_{r} & =\inf _{u \in \Phi^{-1}(r)} I(u) \\
& =\inf _{u \in \Phi^{-1}(r)}\left\{\sum_{t=1}^{2}\left[\rho_{1}(t)\left(\frac{\left|\Delta u_{1}(t)\right|^{2}}{2}+\frac{\left|\Delta u_{1}(t)\right|^{\frac{7}{3}}}{\frac{7}{3}}\right)+\rho_{2}(t)\left(\frac{\left|\Delta u_{2}(t)\right|^{2}}{2}+\frac{\left|\Delta u_{2}(t)\right|^{\frac{5}{2}}}{\frac{5}{2}}\right)\right]\right.
\end{aligned}
$$




$$
\begin{aligned}
& \left.+\sum_{t=1}^{2}\left[\rho_{3}(t)\left|u_{1}(t)\right|^{2}+\rho_{4}(t)\left|u_{2}(t)\right|^{2}\right]\right\} \\
\geqslant & \inf _{u \in \in \Phi^{-1}(r)} \sum_{t=1}^{2}\left[\rho_{3}(t)\left|u_{1}(t)\right|^{2}+\rho_{4}(t)\left|u_{2}(t)\right|^{2}\right] \\
= & \inf _{u \in \Phi^{-1}(r)}\left(\rho_{3}(1)\left|u_{1}(1)\right|^{2}+\rho_{3}(2)\left|u_{1}(2)\right|^{2}+\rho_{4}(1)\left|u_{2}(1)\right|^{2}+\rho_{4}(2)\left|u_{2}(2)\right|^{2}\right) \\
= & \min \left\{\rho_{3}(1), \rho_{3}(2), \rho_{4}(1), \rho_{4}(2)\right\} \sqrt{r},
\end{aligned}
$$

which can be obtained by using the Lagrange multiplier method. By (5.1), (5.2) and the fact that $\Phi(0)=$ $\mathrm{I}(0)=0$, we have

$$
\begin{aligned}
\mu^{*}(I, \Psi, \Phi, r) & =\inf \left\{\frac{\Psi(u)-\gamma+r}{\eta_{r}-I(u)}: u \in E, \Phi(u)<r, I(u)<\eta_{r}\right\} \\
& \leqslant \frac{\Psi(0)-\gamma+r}{\eta_{r}-I(0)}=\frac{\frac{27}{64}+r}{\eta_{r}} \leqslant \frac{\frac{27}{64}+r}{\min \left\{\rho_{3}(1), \rho_{3}(2), \rho_{4}(1), \rho_{4}(2)\right\} \sqrt{r}} .
\end{aligned}
$$

When $\mu>\frac{r^{\frac{1}{4}}}{\min \left\{\rho_{3}(1), \rho_{3}(2), \rho_{4}(1), \rho_{4}(2)\right\}}$, we have

$$
\begin{aligned}
& \inf _{\left.\left.\Phi^{-1}(]-\infty, r\right]\right)}(\mu \mathrm{I}+\Psi) \\
& =\inf _{\left.\left.\Phi^{-1}(]-\infty, r\right]\right)} \mu\left\{\sum_{t=1}^{2}\left[\rho_{1}(\mathrm{t})\left(\frac{\left|\Delta \mathrm{u}_{1}(\mathrm{t})\right|^{2}}{2}+\frac{\left|\Delta \mathrm{u}_{1}(\mathrm{t})\right|^{\frac{7}{3}}}{\frac{7}{3}}\right)+\rho_{2}(\mathrm{t})\left(\frac{\left|\Delta \mathrm{u}_{2}(\mathrm{t})\right|^{2}}{2}+\frac{\left|\Delta \mathrm{u}_{2}(\mathrm{t})\right|^{\frac{5}{2}}}{\frac{5}{2}}\right)\right]\right. \\
& \left.\quad+\sum_{\mathrm{t}=1}^{2}\left[\rho_{3}(\mathrm{t})\left|\mathrm{u}_{1}(\mathrm{t})\right|^{2}+\rho_{4}(\mathrm{t})\left|\mathrm{u}_{2}(\mathrm{t})\right|^{2}\right]\right\}-\sum_{\mathrm{t}=1}^{2}\left(\left|\mathrm{u}_{1}(\mathrm{t})\right|^{3}+\left|\mathrm{u}_{2}(\mathrm{t})\right|^{3}\right)=0,
\end{aligned}
$$

which is obtained when $\mathfrak{u}_{1}(1)=\mathfrak{u}_{1}(2)=\mathfrak{u}_{2}(1)=\mathfrak{u}_{2}(2)=0$. When $\mu>\frac{r^{\frac{1}{4}}}{\min \left\{\rho_{3}(1), \rho_{3}(2), \rho_{4}(1), \rho_{4}(2)\right\}}$, we choose $\mathfrak{u}_{0}: \mathfrak{u}_{1}(1)=\mathfrak{u}_{1}(2)=\mathfrak{u}_{2}(1)=\mathfrak{u}_{2}(2)=\mu\left[\rho_{3}(1)+\rho_{3}(2)+\rho_{4}(1)+\rho_{4}(2)\right]$, then

$$
\begin{aligned}
\left|u_{1}(1)\right|^{4}+\left|u_{1}(2)\right|^{4}+\left|u_{2}(1)\right|^{4}+\left|u_{2}(2)\right|^{4} & =4 \mu^{4}\left[\rho_{3}(1)+\rho_{3}(2)+\rho_{4}(1)+\rho_{4}(2)\right]^{4} \\
& >\frac{4 r\left[\rho_{3}(1)+\rho_{3}(2)+\rho_{4}(1)+\rho_{4}(2)\right]^{4}}{\left(\min \left\{\rho_{3}(1), \rho_{3}(2), \rho_{4}(1), \rho_{4}(2)\right\}\right)^{4}}>r,
\end{aligned}
$$

which implies that $u_{0} \in \Phi^{-1}(] r,+\infty[)$. Therefore, when $\mu>\frac{\max \left\{\frac{\frac{27}{64}+r}{\sqrt{r}}, r^{\frac{1}{4}}\right\}}{\min \left\{\rho_{3}(1), \rho_{3}(2), \rho_{4}(1), \rho_{4}(2)\right\}}$, by (5.3) and the fact that $u_{0} \in \Phi^{-1}(] r,+\infty[)$ we have

$$
\begin{aligned}
\beta(\mu \mathrm{I}+\Psi, \Phi, r) & =\sup _{u \in \Phi^{-1}(] r,+\infty[)} \frac{\mu \mathrm{I}(u)+\Psi(u)-\inf _{\left.\left.\Phi^{-1}(]-\infty, r\right]\right)}(\mu \mathrm{I}+\Psi)}{r-\Phi(u)} \\
& =\sup _{u \in \Phi^{-1}(] r,+\infty[)} \frac{\mu \mathrm{I}(u)+\Psi(u)}{r-\Phi(u)} \\
& \geqslant \frac{\mu I\left(u_{0}\right)+\Psi\left(u_{0}\right)}{r-\Phi\left(u_{0}\right)}=\frac{3 \mu^{3}\left[\rho_{3}(1)+\rho_{3}(2)+\rho_{4}(1)+\rho_{4}(2)\right]^{3}}{4 \mu^{4}\left[\rho_{3}(1)+\rho_{3}(2)+\rho_{4}(1)+\rho_{4}(2)\right]^{4}-r} .
\end{aligned}
$$

Hence, by Theorem 1.2, we obtain that for each $r>0$, for each $\mu>\frac{\max \left\{\frac{\frac{27}{4}+r}{\sqrt{r}}, r^{\frac{1}{4}}\right\}}{\min \left\{\rho_{3}(1), \rho_{3}(2), \rho_{4}(1), \rho_{4}(2)\right\}}$, and for each compact interval $[a, b] \subset] 0, \frac{3 \mu^{3}\left[\rho_{3}(1)+\rho_{3}(2)+\rho_{4}(1)+\rho_{4}(2)\right]^{3}}{4 \mu^{4}\left[\rho_{3}(1)+\rho_{3}(2)+\rho_{4}(1)+\rho_{4}(2)\right]^{4}-r}[$, there exists a number $\rho>0$ with the following property: for every $\lambda \in[a, b]$, there exists $\delta>0$ such that, for each $v \in[0, \delta]$, system (1.1) has at least three 2-periodic solutions in $E$ whose norms are less than $\rho$. 
Example 5.2. We present this example when Theorem 1.4 is applied. Let $N=6$ and $T=4$. Assume that $\phi_{1}(x)=\phi_{3}(x)=|x|^{3} x, \phi_{2}(x)=\phi_{4}(x)=|x| x$. Consider the following nonlinear difference $\left(\phi_{1}, \phi_{2}\right)$ Laplacian system:

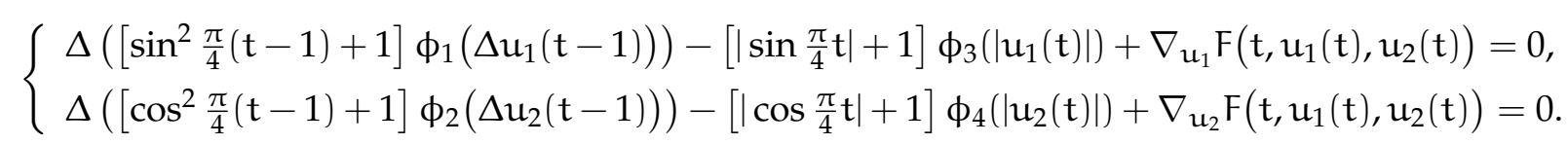

Then $\gamma_{1}(t)=\sin ^{2} \frac{\pi}{4} t+1, \gamma_{2}(t)=\cos ^{2} \frac{\pi}{4} t+1, \gamma_{3}(t)=\left|\sin \frac{\pi}{4} t\right|+1, \gamma_{4}(t)=\left|\cos \frac{\pi}{4} t\right|+1$. Obviously, the conditions $(\gamma)$ and $(\phi)$ hold and $\gamma_{i}, i=1,2,3,4$ are T-periodic $(T=4)$. If we assume that

$$
F\left(t, x_{1}, x_{2}\right)=\left(\left|\sin \frac{\pi}{4} t\right|+1\right)\left|x_{1}\right|^{\frac{3}{2}}+\left(\cos ^{2} \frac{\pi}{4} t+1\right)\left|x_{2}\right|^{2},
$$

then, obviously, $(\mathcal{F} 0),(\mathcal{F} 1)$, and $(\mathcal{F} 2)$ hold and there exists enough small $\delta \in(0,1)$ such that

$$
\begin{aligned}
\mathrm{F}\left(\mathrm{t}, \mathrm{x}_{1}, \mathrm{x}_{2}\right) & =\left(\left|\sin \frac{\pi}{4} \mathrm{t}\right|+1\right)\left|\mathrm{x}_{1}\right|^{\frac{3}{2}}+\left(\cos ^{2} \frac{\pi}{4} \mathrm{t}+1\right)\left|\mathrm{x}_{2}\right|^{2} \\
& \geqslant\left|x_{1}\right|^{\frac{3}{2}}+\left|x_{2}\right|^{2} \geqslant\left|x_{1}\right|^{2}+\left|x_{2}\right|^{\frac{5}{2}}, \quad \forall\left|x_{1}\right|<\delta,\left|x_{2}\right|<\delta .
\end{aligned}
$$

Let $\beta_{1}=2, \beta_{2}=\frac{5}{2}$, and $M_{1}=M_{2}=1$. Then (5.5) implies that (F3) holds. Hence, by Theorem 1.4, we obtained that system (5.4) has at least 48 distinct pairs of 4-periodic solutions.

\section{Conclusion}

In this paper, we obtain system (1.1) has at least three T-periodic solutions by using an abstract critical point theorem due to Ricceri [17], and we present an example which particularly shows that the parameters $\mu$ and $\lambda$ can be estimated. Moreover, we also obtain system (1.5) has 2NT distinct pairs of nonzero T-periodic solutions by using Clark's Theorem, and we also present an example to show that Theorem 1.4 is feasible.

\section{Acknowledgment}

This project is supported by the National Natural Science Foundation of China (No. 11301235).

\section{References}

[1] G. Bonanno, P. Candito, Nonlinear difference equations investigated via critical point methods, Nonlinear Anal., 70 (2009), 3180-3186. 1

[2] P. Candito, N. Giovannelli, Multiple solutions for a discrete boundary value problem involving the p-Laplacian, Comput. Math. Appl., 56 (2008), 959-964.

[3] H.-Y. Deng, X.-Y. Zhang, H. Fang, Existence of periodic solutions for a class of discrete systems with classical or bounded $\left(\phi_{1}, \phi_{2}\right)$-Laplacian, J. Nonlinear Sci. Appl., 10 (2017), 535-559. 1, 1

[4] Z.-M. Guo, J.-S. Yu, Existence of periodic and subharmonic solutions for second-order superlinear difference equations, Sci. China Ser. A, 46 (2003), 506-515.

[5] Z.-M. Guo, J.-S. Yu, The existence of periodic and subharmonic solutions of subquadratic second order difference equations, J. London Math. Soc., 68 (2003), 419-430.

[6] T.-S. He, W.-G. Chen, Periodic solutions of second order discrete convex systems involving the p-Laplaci, Appl. Math. Comput., 206 (2008), 124-132. 1

[7] X.-F. He, P. Chen, Homoclinic solutions for second order discrete p-Laplacian systems, Adv. Difference Equ., 2011 (2011), 16 pages. 1

[8] Y.-K. Li, T.-W. Zhang, Infinitely many periodic solutions for second-order ( $\mathrm{p}, \mathrm{q})$-Laplacian differential systems, Nonlinear Anal., 74 (2011), 5215-5221. 1

[9] X.-Y. Lin, X.-H. Tang, Existence of infinitely many homoclinic orbits in discrete Hamiltonian systems, J. Math. Anal. Appl., 373 (2011), 59-72. 1

[10] J. Q. Liu, A generalized saddle point theorem, J. Differential Equations, 82 (1989), 372-385. 1 
[11] J. Mawhin, Periodic solutions of second order nonlinear difference systems with $\phi$-Laplacian: a variational approach, Nonlinear Anal., 75 (2012), 4672-4687. 1.1, 1, 1, 3

[12] J. Mawhin, Periodic solutions of second order Lagrangian difference systems with bounded or singular $\phi$-Laplacian and periodic potential, Discrete Contin. Dyn. Syst. Ser. S, 6 (2013), 1065-1076. 1, 1, 1

[13] J. Mawhin, M. Willem, Critical point theory and Hamiltonian systems, Applied Mathematical Sciences, SpringerVerlag, New York, (1989). 1

[14] D. Paşca, Periodic solutions of a class of nonautonomous second order differential systems with (q, p)-Laplacian, Bull. Belg. Math. Soc. Simon Stevin, 17 (2010), 841-850. 1

[15] D. Paşca, C.-L. Tang, Some existence results on periodic solutions of nonautonomous second-order differential systems with (q, p)-Laplacian, Appl. Math. Lett., 23 (2010), 246-251. 1

[16] P. H. Rabinowitz, Minimax methods in critical point theory with applications to differential equations, CBMS Regional Conference Series in Mathematics, Published for the Conference Board of the Mathematical Sciences, Washington, DC; by the American Mathematical Society, Providence, RI, (1986). 4.2

[17] B. Ricceri, A further refinement of a three critical points theorem, Nonlinear Anal., 74 (2011), 7446-7454. 1, 1, 2, 2.3, 5

[18] M. Schechter, Minimax systems and critical point theory, Birkhäuser Boston, Inc., Boston, MA, (2009). 1

[19] X. H. Tang, X.-Y. Lin, Existence and multiplicity of homoclinic solutions for second-order discrete Hamiltonian systems with subquadratic potential, J. Difference Equ. Appl., 17 (2011), 1617-1634. 1, 1

[20] X. H. Tang, X.-Y. Zhang, Periodic solutions for second-order discrete Hamiltonian systems, J. Difference Equ. Appl., 17 (2011), 1413-1430.

[21] Y. Wang, X.-Y. Zhang, Multiple periodic solutions for a class of nonlinear difference systems with classical or bounded $\left(\phi_{1}, \phi_{2}\right)$-Laplacian, Adv. Difference Equ., 2014 (2014), 33 pages. 1, 1, 2.1

[22] Y.-F. Xue, C.-L. Tang, Existence of a periodic solution for subquadratic second-order discrete Hamiltonian system, Nonlinear Anal., 67 (2007), 2072-2080. 1

[23] X.-X. Yang, H.-B. Chen, Periodic solutions for autonomous (q, p)-Laplacian system with impulsive effects, J. Appl. Math., 2011 (2011), 19 pages. 1

[24] X.-X. Yang, H.-B. Chen, Periodic solutions for a nonlinear ( $q, p)$-Laplacian dynamical system with impulsive effects, J. Appl. Math. Comput., 40 (2012), 607-625. 1

[25] E. Zeidler, Nonlinear functional analysis and its applications, II/B, Nonlinear monotone operators, Translated from the German by the author and Leo F. Boron, Springer-Verlag, New York, (1990). 3

[26] X.-Y. Zhang, Notes on periodic solutions for a nonlinear discrete system involving the p-Laplacian, Bull. Malays. Math. Sci. Soc., 37 (2014), 499-509. 1

[27] X.-Y. Zhang, X.-H. Tang, Existence of solutions for a nonlinear discrete system involving the p-Laplacian, Appl. Math., 57 (2012), 11-30.

[28] Q.-F. Zhang, X. H. Tang, Q.-M. Zhang, Existence of periodic solutions for a class of discrete Hamiltonian systems, Discrete Dyn. Nat. Soc., 2011 (2011), 14 pages.

[29] X.-Y. Zhang, Y. Wang, Homoclinic solutions for a class of nonlinear difference systems with classical $\left(\varphi_{1}, \varphi_{2}\right)$-Laplacian, Adv. Difference Equ., 2015 (2015), 24 pages. 1, 1, 1

[30] Z. Zhou, J.-S. Yu, Z.-M. Guo, Periodic solutions of higher-dimensional discrete systems, Proc. Roy. Soc. Edinburgh Sect. A, 134 (2004), 1013-1022. 1 